Mens

revue d'histoire intellectuelle de l'Amérique française

\title{
Peinture et territoire en dialogue. Regard de Paul-Émile Borduas sur l'Amérique
}

\section{Louise Vigneault}

Volume 10, numéro 1, automne 2009

URI : https://id.erudit.org/iderudit/1023159ar

DOI : https://doi.org/10.7202/1023159ar

Aller au sommaire du numéro

Éditeur(s)

Centre de recherche en civilisation canadienne-française

ISSN

1492-8647 (imprimé)

1927-9299 (numérique)

Découvrir la revue

Citer cet article

Vigneault, L. (2009). Peinture et territoire en dialogue. Regard de Paul-Émile Borduas sur l'Amérique. Mens, 10(1), 51-93. https://doi.org/10.7202/1023159ar
Résumé de l'article

Cet article propose d'analyser la manière dont Paul-Émile Borduas a peu à peu intégré, au cours de ses exils aux États-Unis et en France, les fondements culturels et l'imaginaire spatial du continent nord-américain, comment ces modèles ont pris forme et se sont exprimés sur le plan esthétique et plastique. L'examen des œuvres de la période tardive (1953-1959), en fonction de la vision que l'artiste aurait entretenue de sa position et de sa condition dans l'espace géographique et social, a permis également de compléter les analyses formalistes et sémiotiques menées jusqu'à ce jour. 


\title{
Peinture et territoire en dialogue. Regard de Paul-Émile Borduas sur l'Amérique
}

\author{
Louise Vigneault \\ Département d'histoire de l'art \\ et d'études cinématographiques \\ Université de Montréal
}

\section{Résumé}

Cet article propose d'analyser la manière dont Paul-Émile Borduas a peu à peu intégré, au cours de ses exils aux États-Unis et en France, les fondements culturels et l'imaginaire spatial du continent nordaméricain, comment ces modèles ont pris forme et se sont exprimés sur le plan esthétique et plastique. L'examen des œuvres de la période tardive (1953-1959), en fonction de la vision que l'artiste aurait entretenue de sa position et de sa condition dans l'espace géographique et social, a permis également de compléter les analyses formalistes et sémiotiques menées jusqu’à ce jour.

\section{Abstract}

This article explores the manner in which Paul-Emile Borduas gradually incorporated the cultural foundations and the spatial imaginary of the North American continent into his work during his self-exile in the United States and France, and how these models took form and expressed themselves esthetically and plastically. An examination of Borduas' later work (1953-1959) as a function of the vision that he held of his position and of his geographic and social condition completes previously published formalist and semiotic analyses. 
Lorsqu'au cours des années 1940, les artistes nord-américains ont été confrontés sur leur propre terrain à la présence des avantgardes européennes en exil, les sentiments qu'ils éprouvaient alors à leur endroit avaient pour effet d'éveiller le besoin d'outrepasser leur position périphérique, de définir une modernité artistique solide, susceptible d'être reconnue sur le plan international, mais aussi, de cerner les particularités de leur culture dont les traits demeuraient encore imprécis. Au cours de cette période charnière qui a engendré un bouleversement de l'hégémonie artistique occidentale, Paul-Émile Borduas reconnaît progressivement l'importance des modèles artistiques états-uniens dans le développement de la modernité picturale. Cet éveil s'effectue au cours de ses séjours aux États-Unis (1953-1955) et en France (1955-1960), des expériences qui ont alors pour effet d'éprouver sa perception de l'imaginaire nord-américain de l'espace, et ce, malgré la méfiance que le milieu artistique montréalais entretient en général à l'endroit de la culture états-unienne et de ses productions. L'examen des expériences extrafrontalières de Borduas, partagées entre l'essor artistique états-unien et le réseau européen, permettra de mieux saisir comment l'artiste percevait sa propre situation dans l'environnement physique et social, ainsi que la manière dont cette vision aurait orienté sa démarche et ses choix esthétiques. Dans une plus large mesure, cette étude de cas vise à mieux saisir le rôle de l'imaginaire spatial continental dans le processus de définition de la modernité artistique québécoise.

En cherchant à relativiser les rapports bilatéraux qu'entretenaient les créateurs québécois et états-uniens du XIX siècle, et les traits proprement américains attribués à leurs productions, David Karel avait recouru au schéma triangulaire, de manière à rendre justice à la présence intermédiaire des intervenants européens qui avaient orchestré en partie les développements stylistiques'. Bien que cette initiative louable ait participé aux premiers comptes rendus

${ }^{1}$ David Karel, «L'expérience continentale de l'art québécois au XIX siècle», dans Gérard Bouchard et Yvan Lamonde, dir., Québécois et Américains. La culture québécoise aux XIX et XXX siècles, Montréal, Fides, 1993, p. 197-225. 
scientifiques consacrés à la question de l'américanité, elle méritait à notre avis d'être complétée par l'examen de l'imaginaire spatial et des moteurs de création qui ont forgé les cultures visuelles. Les idéaux humanistes et universalistes que caressaient les artistes, ainsi que la mobilité croissante qui leur permettait de «tester » les possibilités de leur concrétisation, ont d'ailleurs fait en sorte de complexifier cette triangularité d'échanges. Dans ce contexte, les anciens schèmes dichotomiques qui avaient opposé jusque-là les idéaux modernes à toute forme d'appartenance ou d'ancrage géographique se trouvaient ébranlés.

\section{Définir la culture américaine}

En 1983, Serge Guilbaut publiait Comment New York vola l'idée d'art moderne dans lequel était examiné le rôle de certains événements de l'histoire états-unienne dans la consolidation de sa culture artistique, ainsi que les échos idéologiques et les circonstances sociopolitiques qui ont mené au transfert de l'hégémonie artistique de Paris à New York: montée du nationalisme, essor économique, création d'une culture issue de la classe moyenne, d'un marché et d'un mécénat libérés de la suprématie parisienne ${ }^{2}$. Cette étude avait eu le mérite d'insister sur la genèse du phénomène plutôt que sur son triomphe ${ }^{3}$, et de révéler la manière dont l'avant-garde américaine avait été en mesure de conjuguer l'exercice de rattrapage des critères artistiques européens et la mise en place d'une pratique picturale solidement ancrée dans la culture locale. Le défi avait été relevé notamment grâce à l'efficacité de l'appareil mythologique qui avait comme effet de résoudre

\footnotetext{
2 Serge Guilbaut, dir., Reconstructing Modernism: Art in New York, Paris, and Montreal (1945-1964), Cambridge, MIT Press, 1990, 418 p.; Comment New York vola l'idée d'art moderne, traduit de l'anglais par Catherine Fraixe, Paris, Hachette Littératures, 1996 [1983], $342 \mathrm{p}$.

${ }^{3}$ L'auteur rapporte notamment le discours de Sam Hunter, Modern American Painting and Sculpture, New York, Dell, 1959, 256 p.; Irving Sandler, Triumph of American Painting, New York, Praeger Publishers, 1970, 301 p. et Barbara Rose, American Art Since 1900, New York, Praeger Publishers, 1967, 320 p.
} 
certains conflits idéologiques, et grâce à l'adaptation des idéaux modernes à la condition américaine et à ses impératifs de développement $t^{4}$. Tout en maintenant un dialogue avec la tradition européenne, le milieu culturel avait été en mesure, par exemple, d'assumer certaines contradictions, comme celle de se montrer progressiste sans adhérer à des aspirations politiques révolutionnaires ou de gauche. C'est ainsi que les valeurs modernes se sont trouvées parfaitement intégrées aux mailles de son tissu social, culturel et politique. Les événements de la Seconde Guerre, mais également les luttes menées par les intellectuels de part et d'autre de l'Atlantique pour la défense de la culture moderne contre le fascisme, puis le communisme, ont contribué par la suite à faire basculer les dominos en faveur de l'american democratic way of life, au détriment de la capitale française qui avait été jusque-là réputée pour son contexte de liberté d'idées et de mœurs ${ }^{5}$. Guilbaut souligne toutefois la contradiction que renferme cette prise de position:

Le modernisme rejeté par le fascisme était aux U.S.A. confondu et assimilé avec une définition large et abstraite de la culture. Ainsi, la grande presse défendait, sans le savoir, le concept de modernité avec toutes les ambiguïtés et contradictions que cela comportait. Ce modernisme, qui n'avait jamais réussi auparavant à prendre racine aux U.S.A., s'introduisait par une porte dérobée, par erreur en quelque sorte, dans la conscience nationale ${ }^{6}$.

Cette modernité ne pouvait pourtant produire un art valable au sein de la culture nationale qu'en se libérant de l'héritage européen et en assumant ses positions spécifiques, malgré ses maladresses apparentes. Il devenait nécessaire, du même coup, de reconsidérer la place que cette culture occupait dans la pensée internationaliste. En 1940, une table ronde organisée à l'Université de Chicago, et retransmise à la radio, révèle le rôle que les instances politiques

\footnotetext{
${ }^{4}$ Guilbaut, dir., Comment New York vola l'idée d'art moderne, p. 73-74.

${ }^{5}$ Ibid., p. 64-71.

${ }^{6}$ Ibid., p. 71.
} 
entendent accorder à l'art. À cette occasion, le critique littéraire Clifton Fadiman déclare:

Nous sommes parvenus à un seuil critique de la vie de notre pays. Le pays des pionniers, c'est fini; aujourd'hui, c'est une véritable civilisation que nous allons développer. [...] l'art est un moyen d'unifier le peuple.[...] Dans ce pays, [l'unité] ne signifie pas, comme dans d'autres pays, se laisser enrégimenter. Pour nous, ce sont des gens qui se mettent d'accord, ou essaient de se mettre d'accord, plus ou moins sincèrement, sur quelques points fondamentaux, et qui n'arrivent pas à se mettre d'accord sur beaucoup d'autres'.

Au Québec, le courant personnaliste, qui vise notamment à contrer le "malaise civilisationnel », permet de réconcilier les implications spirituelle et sociale, grâce à un solide enracinement dans le réel et à un retour aux sources de l'Être ${ }^{8}$. Pour Borduas et ses collègues, cette éthique constitue, pour en temps, un compromis efficace entre le respect des besoins essentiels de l'individu, le maintien d'une équité au sein de la collectivité moderne, et l'engagement spirituel et moral de ses membres. Le renforcement de l'opposition entre les forces progressistes et conservatrices poussera toutefois Borduas à abandonner tout modèle idéologique et à endosser une position anarchiste qu'il considère comme la "seule forme sociale ouverte à la multitude des possibilités des réalisations individuelles ${ }^{9}$ ". Cette réclamation radicale d'autonomie face aux institutions prendra diverses formes, dont celle de l'exil qui, malgré les ruptures qu'elle engendre, lui offrira de nouvelles perspectives susceptibles de réconcilier les conflits qui le tenaillent.

\footnotetext{
${ }^{7}$ Table ronde réunissant Eleanor Roosevelt, Fadiman, le bibliothécaire du Congrès A. MacLeish, et le sociologue Louis Wirth. Retransmis par la NBC, le 24 novembre 1940. (Traduction de l'auteure.)

${ }^{8}$ Voir à ce sujet E.-Martin Meunier et Jean-Philippe Warren, Sortir de la "Grande noirceur». L'horizon personnaliste de la Révolution tranquille, Sillery, Septentrion, 2002, 207 p.

"Paul-Émile Borduas, "Refus global ", Refus global et autres écrits, édition préparée et présentée par André-G. Bourassa et Gilles Lapointe, Montréal, L'Hexagone, 1990,301 p.
} 
L'analyse des fondements culturels qui, en Amérique, ont assuré le maintien d'un équilibre entre individualisme et collectivisme, entre matérialisme et spiritualité, permet de saisir la manière dont les artistes canadiens et états-uniens en sont venus à représenter leur « modernité culturelle", au-delà des catégorisations opposant les tenants de la figuration et de l'abstraction, de l'expression et du synthétisme ${ }^{10}$, et au-delà de la prétention américaine à englober l'expérience moderne. Un bref détour historique aux origines de la colonisation révèle, par exemple, qu'en dépit de son absence de mémoire historique substantielle, le territoire nord-américain a été exploité comme une entité culturellement chargée, grâce à la fusion des réalités spatiale et temporelle. Cette articulation trouvait notamment sa source dans les déplacements successifs vers la frontière, zone liminale et mouvante, qui délimite l'inconnu et le connu, la nature et la culture, et convie à une confrontation avec l'altérité et avec soi-même ${ }^{11}$. Le fonctionnement nomade, qui caractérise les expériences de transit, d'exil ou d'exode, permettait à ce titre d'échapper aux contraintes sociales et politiques, et offrait une possible amélioration des conditions de vie $^{12}$. Forts de ce potentiel de renouvellement, les pionniers nordaméricains ne se seraient donc pas engagés dans une dynamique d'errance, comme c'est le cas en Europe des groupes marginalisés, mais dans un mouvement renouvelable d'exploration et d'expansion, qui a contribué à combler l'absence de mémoire et d'historicité. Ces

\footnotetext{
${ }^{10}$ À ce titre, la conservatrice Bernice Rose concluait, en 1980, à propos de l'École de New York: "On passe ainsi de la métaphore de la "scène américaine" et des récits des images symboliques/mythologiques des années trente et quarante à la subjectivité et à l'abstraction de l'œuvre de la maturité, avec son désir d'universalité - de "refléter l'expérience de notre temps". Il faut se demander ce qui se reflète là de la société en général et de la société américaine en particulier. " Jackson Pollock: Drawing into Painting, New York, MoMA, 1980, p. 23. Traduction de Catherine Fraixe tirée de Serge Guilbaut, Comment New York vola l'idée d'art moderne, p. 25.

${ }^{11}$ Les données qui suivent ont été explicitées dans notre article "Le pionnier : acteur de la frontière ", dans Bernard Andrès et Gérard Bouchard, dir., Mythes et sociétés des Amériques, Montréal, Québec/Amérique, 2007, p. 275-311.

${ }^{12}$ Voir à ce sujet Jean Morency, Le mythe américain dans les fictions d'Amérique: de Washington Irving à Jacques Poulin, Québec, Nuit Blanche, 1994, 258 p.
} 
expériences de transit et de confrontation des espaces inconnus ont engendré des croisements significatifs dans la construction des cultures et de l'imaginaire spatial des communautés, comme l'ont notamment analysé Jean Morency et Gérard Bouchard ${ }^{13}$. Dans ce contexte, la thématique du paysage, qui a d'abord monopolisé les productions picturales des XVIII et XIX ${ }^{e}$ siècles, a permis aux artistes d'insuffler au territoire une charge culturelle significative et distincte. Les artistes de la Hudson River School, ceux des campagnes d'exploration de l'Ouest, ainsi que les photographes et peintres canadiens qui ont témoigné du développement du territoire, ont tenté en premier lieu d'exprimer les effets de l'environnement sur les populations. Réinterprétée en fonction de l'expérience de la frontière, la réalité territoriale était représentée comme une zone de mise en tension des forces fondamentales, attribuant ainsi à l'héritage romantique un tout nouveau sens. Cette réorientation des modèles européens motivait, par la suite, les artistes à se détacher progressivement des rhétoriques traditionnelles afin d'investir un rapport plus direct à la réalité, à recourir à une approche ontologique et heuristique de l'espace, laquelle a engendré un véritable retour aux sources du langage pictural. Au Québec toutefois, les difficultés qu'ont éprouvées les factions progressistes à mettre de l'avant les valeurs de renouveau et d'ouverture les ont forcés le plus souvent à reconsidérer leur appartenance géosociétale, et à chercher des modèles de représentation en périphérie.

$\mathrm{Si}$, dans le contexte moderne, le territoire a été le plus souvent perçu comme un ancrage duquel les artistes ont tenté de s'arracher, afin d'adhérer aux idéaux universalistes, l'avant-garde nord-américaine l'aurait abordé comme un lieu de confrontation et de réflexion sur la condition humaine. Marqués par le trauma de la crise et du second conflit mondial, les artistes ont cherché à se détacher des référents locaux ou sociaux, afin d'exprimer l'expérience générique de l'humanité moderne confrontée à sa destinée. En raison des afflictions que

${ }^{13}$ Ibid.; Gérard Bouchard, Genèse des nations et cultures du Nouveau Monde, Montréal, Boréal, 2000, 503 p. 
partageaient les Nord-Américains, natifs ou migrants, par rapport aux années de crise, à la montée du fascisme et à la recrudescence des mouvements de déplacement, les productions artistiques se sont trouvées imprégnées des mythologies fondatrices revues et corrigées qui permettaient d'exprimer cette "tragédie existentielle ", comme l'a exprimée notamment le peintre d'origine lettonienne Mark Rothko, au début des années 1940 :

[l'émotionnalisme universel] en rapport avec l'individu ne se trouve que dans l'émotionnalité tragique. [...] la douleur, la frustration et la peur de la mort semblent être le liant le plus constant entre les êtres humains, et nous savons qu'un ennemi commun réussit beaucoup mieux à souder les énergies et bien plus efficacement à effacer les singularités qu'une fin positive commune ${ }^{14}$.

Ce croisement des imaginaires de rupture et de transit a d'ailleurs engendré certaines synchronies dans le développement de l'art moderne, au Canada et aux États-Unis. À ce titre, on constate par exemple que, sans avoir entretenu des contacts significatifs, les automatistes montréalais et les expressionnistes abstraits new-yorkais ont réinterprété sensiblement de la même manière les expériences surréalistes de l'automatisme surrationnel ou mécanique. Au cours des années 1940, les expressionnistes abstraits (Arshile Gorky, William Baziotes, Jackson Pollock, Willem De Kooning, Hans Hofmann, Adolph Gottlieb, pour ne nommer qu'eux) ${ }^{15}$, qui investissent comme leurs collègues européens un espace-écran destiné à recevoir l'expression spontanée des pulsions inconscientes, éprouvent toutefois des réserves à l'égard de la notion freudienne du désir qui est ramenée, selon eux, à des considérations trop strictement intimes, à des " tragédies personnelles ${ }^{16}$. Leur moteur de création consiste d'ailleurs

${ }^{14}$ Mark Rothko. La Réalité de l'artiste, traduit de l'anglais par Pierre-Emmanuel Dauzat, Paris, Flammarion, 2004, p. 40. La majeure partie du texte aurait été rédigée en 1940-1941.

${ }^{15}$ Le lecteur consultera le site suivant, pour une liste exhaustive des artistes, ainsi que des exemples d'œuvres: http://www.artlex.com/ArtLex/a/abstractexpr.html.

${ }^{16}$ Dore Ashton, L'École de New York. Histoire d'un mouvement, Paris, Hazan, 1992 [1973], $265 \mathrm{p}$. 
moins en une dynamique de révolte et de réaction face à la collectivité et au passé, comme le vivent leurs collègues européens, qu'en un acte primaire d'existence et d'affirmation ${ }^{17}$. Se tournant plus spontanément vers les modèles mythologiques ou archétypaux, qu'ils prennent soin d'adapter à la réalité américaine contemporaine, les artistes font davantage appel à l'inconscient dans le processus même, en misant sur la dimension performative et en maintenant un rapport de proximité avec leur environnement. Libérées des intermédiaires rhétoriques qui marquent une distance entre l'objet et son interprétation, les œuvres présentent en somme une interpénétration de l'espace réel et de l'espace imaginé.

Bien que les artistes européens cherchent également à libérer l'activité artistique de ses références traditionnelles à des champs parallèles - philosophique, littéraire, etc. - , leur exercice de déconstruction se trouve à contenir intrinsèquement, à travers les marques de leur réaction, ces mêmes références contestées, tandis que le langage choisi pour exprimer cette réaction conserve le plus souvent une rhétorique complexe, hautement intellectualisée, comme en témoignent notamment les initiatives des surréalistes. Dans ce contexte, la déconstruction des anciens schèmes aurait pris le pas sur un réel renouvellement du langage artistique et de ses codes. Chez les Nord-Américains, en revanche, l'expression individuelle se voit plutôt guidée par une expérience pragmatique et un rapport sans détour avec le réel objectif, que les surréalistes ont pourtant choisi eux aussi d'investir afin de servir leurs désirs enfouis, mais qui s'inscrivent plus spécifiquement, en Amérique, dans le sillage des valeurs modernes issues des mythologies fondatrices, revues à la lumière des "nouvelles frontières" à conquérir. À ce titre, Margit Rowell soutient qu'en éliminant l'histoire, les "peintres d'action" ont instauré un espace «mythique » dont le sens et la pertinence ont

${ }^{17}$ Comme le souligne Margit Rowell, «il ne leur incombait pas de fuir mais d'agir " (La peinture, le geste et l'action, Paris, Klincksieck, 1972, p. 24). 
été réitérés par la dimension rituelle de l'acte artistique ${ }^{18}$. L'établissement d'une filiation entre la démarche de l'artiste et celle des générations d'immigrants et de défricheurs a été souligné par bon nombre de critiques, d'historiens et de théoriciens ${ }^{19}$. Dore Ashton, par exemple, rappelle l'identification des artistes à la condition pragmatique des ouvriers et des pionniers: "Les expressionnistes abstraits se faisaient souvent gloire d'être nés dans la classe ouvrière, et certains d'entre eux saisissaient les occasions de se distinguer dans la sphère des activités matérielles - le genre de vrai travail qu'ils associaient à l'esprit pionnier des bûcherons et des défricheurs ${ }^{20}$. "Rowell insiste pour sa part sur la dimension performative de l'ascendance mythique: «Le pionnier se trouva responsable de lui-même et de ses actes; l'action fut sa seule expression, l'événement immédiat la seule réalité contre laquelle se mesurer $^{21}$. " Bien qu'elles n'aient pas été considérées par la critique formaliste comme une composante significative de l'œuvre et une partie intégrante du résultat, les dimensions performative, empirique et matérielle des productions auraient permis à l'avant-garde de contourner les oppositions traditionnelles (figuration/abstraction, expression/géométrisation), et d'inaugurer un nouveau rapport entre la forme et l'espace. Si le contexte socioculturel états-unien a encouragé cette entreprise, l'avant-garde montréalaise a rencontré des résistances majeures, attribuables en partie aux contraintes conservatrices, mais aussi à la méfiance des artistes à l'égard des fondements culturels continentaux en lesquels peu se reconnaissaient, et à leur fidélité aux modèles culturels français qui continuaient de faire autorité.

\footnotetext{
${ }^{18}$ Ibid., p. 17.

${ }^{19}$ Voir notamment Harold Rosenberg, Tradition of the New, New York et Toronto, McGraw Hill, 1960, 285 p.; Margit Rowell, La peinture, le geste, l'action, Paris, Klincksieck, 1972, 162 p.; Ellen G. Landau, Jackson Pollock, New York, Abrams, 1989, 283 p.; Kirk Varnedoe et Pepe Karmel, Jackson Pollock, New York, The Museum of Modern Art, 1998, 336 p.

${ }^{20}$ Ashton, L'École de New York. Histoire d'un mouvement, p. 15.

${ }^{21}$ Rowell, La peinture, le geste, l'action, p. 17.
} 
Dans le collectif Québécois et Américains. La culture québécoise aux XIX et XX siècles (1995), Gérard Bouchard et Yvan Lamonde ont exploré la dimension culturelle de la réalité continentale, en tentant d'établir une première mise au point de la perception qu'a entretenue la communauté québécoise de l'expérience "amériquaine ", au-delà de la stricte question des relations et des influences. Dans le cadre de cette étude, François-Marc Gagnon a examiné la manière dont l'avantgarde montréalaise a abandonné les paramètres européens au profit des modèles états-uniens, ainsi que la vision qu'elle a entretenue de l'American-Type Painting ${ }^{22}$. L'auteur y stipule que la définition d'une américanité picturale serait d'abord passée par une prise de conscience de la peinture états-unienne et de son importance dans le contexte de l'après-guerre. Un examen des circonstances qui ont mené Borduas à découvrir la peinture états-unienne, et des perceptions qu'il aurait entretenues de sa situation d'exilé dans les différents environnements, nous permettra de saisir un peu mieux la complexité de ses motivations, et les tournants esthétiques qui ont marqué les dernières années de sa carrière.

\section{Borduas et les États-Unis}

Le premier séjour de Borduas aux États-Unis remonte à 1928. Suivant les conseils d'Ozias Leduc, il se rend d'abord à Boston ${ }^{23}$, afin d'y admirer notamment Puvis de Chavannes, puis au Metropolitain Museum de New York dans le but de découvrir non pas l'art nordaméricain contemporain, mais la modernité artistique européenne. Au cours de sa deuxième visite en 1943, Borduas tente alors, sans succès, de prendre contact avec André Breton et ses collègues, dont

\footnotetext{
${ }^{22}$ François-Marc Gagnon, "La première expérience de la peinture américaine abstraite (les années 1950)", dans Gérard Bouchard et Yvan Lamonde, dir., Québécois et Américains. La culture québécoise auX XIX et XXX siècles, Montréal, Fides, 1995, p. 258.

${ }^{23}$ Rapporté par François-Marc Gagnon, Borduas and America let l'Amérique, The Vancouver Art Gallery, Musées Nationaux du Canada, 1978, p. 27. Musée d'art contemporain de Montréal. Notes biographiques (T261).
} 
la présence rayonne au sein de l'élite culturelle new-yorkaise depuis leur exil, et de renforcer les liens entre le mouvement parisien et le groupe de Montréal ${ }^{24}$. L'avant-garde américaine ne connaît pas, au cours de cette période, une diffusion et une reconnaissance suffisamment importantes pour qu'il puisse en juger. Au cours des années 1940, Borduas expérimente la technique de l'automatisme psychique. Les touches picturales exécutées spontanément produisent des motifs évocateurs qui évoluent dans un espace lyrique, lequel est libéré des lois de la gravité, tout en continuant de respecter l'effet creusant de la perspective ${ }^{25}$. Ces œuvres, qui engendrent un éclatement des structures traditionnelles et un affranchissement des moyens plastiques, suggèrent alors, par extension symbolique, la libération de l'artiste des cadres sociaux et moraux qu'il considère aliénants. Pour cette raison, Borduas maintient, jusqu'au début des années 1950, que l'expression d'un contenu intime refoulé constitue la finalité première des œuvres, et que l'intervention de la conscience dans le processus créatif doit être minimisée, en raison de son adéquation à la rationalité, à la censure, aux «intentions " qui constituent un frein à l'expression de l'inconscient, au plein épanouissement de l'individu. En cela, il se démarque à la fois des surréalistes et des expressionnistes abstraits qui tiennent à assurer un équilibre entre les décharges

${ }^{24}$ Ibid., p. 28. Borduas bénéficie également d'informations sur les avant-gardes européennes de passage à New York par l'intermédiaire de Louise Renaud, qui avait été engagée par Pierre Matisse comme gouvernante, et de Françoise Sullivan et Jeanne Renaud qui s'étaient installées à New York pour étudier la danse moderne. François-Marc Gagnon, "New York as Seen from Montreal by Paul-Emile Borduas and the Automatists ", dans Guilbaut, dir., Reconstructing Modernism: Art in New York, Paris, and Montreal (1945-1964), p. 133.

${ }^{25}$ À ce propos, Thérèse Martin explique: "Ces tableaux récupéraient les normes du paysage pour créer un champ visuel infini qui englobait une multitude d'objets dans son atmosphère unificatrice. La lisibilité était obtenue du fait que certains procédés classiques: toile de fond, figures principales en gros plan étaient conservées " ( $\mathrm{La}$ structure spatiale de l'œuvre new-yorkaise de Paul-Émile Borduas (1953-1955) ", p. 13). Voir également Nathalie Clerk, La peinture de Borduas de 1941 à 1948: en quoi reste-t-elle encore attachée à la figuration?, Mémoire de maitrise (histoire de l'art), Université de Montréal, 1974, 201 p. 
spontanées de l'inconscient et l'intervention structurante de la conscience. Vers 1947, deux de ses anciens étudiants de l'École du meuble tentent pourtant de repousser les limites de l'automatisme. Les œuvres de Jean-Paul Riopelle (Ontario, 1947-48; Piège, 1948; Boisé, 1949) et de Marcel Barbeau (Le tumulte de la mâchoire crispée, $1946^{26}$; Forêt vierge, 1948) présentent alors une structure enchevêtrée de lignes ou de taches qui a pour effet d'éliminer l'illusion de profondeur et de ramener le regard en surface. Réduites à l'essentiel et multipliées, les composantes tendent également à perdre leur charge émotionnelle, et à devenir de purs signes qui témoignent de la présence physique de l'artiste. Ce type de composition déhiérarchisée (all-over) rejoint les productions que réalise Jackson Pollock à la même époque. Face aux œuvres de Riopelle et de Barbeau, Borduas manifeste d'abord des réserves, critiquant notamment l'aspect homogène et décoratif de cet exercice qu'il considère comme régressif et insignifiant, en raison notamment de l'abandon du contenu expressif. Dans ses "Commentaires sur les mots courants" (1948), il explique que les motifs "sont peu révélateurs de la personnalité de leur auteur ${ }^{27}$ ", révélant par conséquent son incapacité de détecter le potentiel de développement de l'automatisme, en fonction des particularismes de l'expérience spatiale américaine.

Son ambition de s'installer sur la Côte-Est américaine, de 1953 à 1955, était motivée, selon Gagnon, par la possibilité d'y voir ses œuvres exposées et vendues, et d'y trouver l'ouverture idéologique et culturelle qui lui avait cruellement manqué en sol québécois ${ }^{28}$. Ce voyage doit alors constituer la première étape d'un périple que Borduas envisage comme suit: deux ans à New York, un temps indéterminé

${ }^{26}$ L'œuvre de Barbeau est disponible dans le site suivant: http://www.davidrumsey. com/amico/amico1221280-97572.html.

${ }^{27}$ Paul-Émile Borduas, "Commentaires sur les mots courants", 1948, Écrits I, édition critique par André-G. Bourassa, Jean Fisette et Gilles Lapointe, Montréal, Les Presses de l'Université de Montréal, Université du Québec à Montréal, 1987, p. 302.

${ }^{28}$ Ibid., p. 139. 
à Paris, un an à Londres, puis un séjour à Tokyo ${ }^{29}$. Cette pulsion de départ se justifie par sa crainte de subir un étouffement culturel, par le besoin d'acquérir la reconnaissance qu'on lui refusait au Québec et de briser la "fatale destinée » de sa communauté, comme il l'avait exprimé plus tôt dans le manifeste automatiste. Mais ce réflexe réactif contre son milieu côtoie aussi celui d'atteindre un univers éloigné, en marge, une altérité en laquelle il pourrait se reconnaître (comme il se reconnaissait dans les enfants et ses étudiants), qui le transformerait radicalement et contribuerait à nourrir son idéal de retour aux sources et de renouvellement intégral de la pratique artistique ${ }^{30}$. Dès les années 1930, il avait d'ailleurs caressé le rêve d'émigrer, comme Gauguin, à Tahiti, dans les Nouvelles-Hébrides ou en Amérique du $\mathrm{Sud}^{31}$. Les circonstances favorables n'étaient toutefois pas au rendezvous. Une vingtaine d'années plus tard, les conséquences de la publication du Refus global ravivent de nouveau l'appel de l'exil. Endeuillé par la détérioration de ses liens familiaux, celui que Guilbaut avait baptisé le "triste vagabond de la modernité ${ }^{32}$ " prend alors la difficile décision de rompre avec ses origines.

À l'été 1953, Borduas s'installe d'abord à Provincetown, où travaille une colonie d'artistes - comme Hans Hofmann, qui y tient

${ }^{29}$ Lettre de Borduas à Fernand Leduc et Thérèse Renaud, Saint-Hilaire-Est, 20 juin 1952. Fonds privé. Écrits II, édition critique par André-G. Bourassa et Gilles Lapointe, Montréal, Les Presses de l'Université de Montréal, Université du Québec à Montréal, 1997, t. I, p. 484.

${ }^{30}$ Voir à ce sujet Louise Vigneault, Identité et modernité dans l'art au Québec. Borduas, Sullivan, Riopelle, Montréal, Hurtubise HMH/Les Cahiers du Québec, 2002, p. 126-133.

${ }^{31}$ François-Marc Gagnon, "La peinture des années trente au Québec », The Journal of Canadian Art History = Annales d'histoire de l'art canadien, vol. 3, $\mathrm{n}^{\text {os }} 1-2$ (automne 1976), p. 17.

32 Serge Guilbault, "Introduction », dans Guilbaut, dir., Reconstructing Modernism: Art in New York, Paris, and Montreal, p. xIIr. Voir notamment Pierre Gauvreau, "Borduas et le déracinement des peintres canadiens ", Le Journal musical canadien, novembre 1954, p. 6; Jean-Pierre Denis, "L'exil au temps des automatistes", dans Lise Gauvin, dir., Les automatistes à Paris, actes d'un colloque, Montréal, Les 400 coups, 2000, p. 79-92. 
une école -, mais entretient peu de contacts avec elle, préférant s'enfermer dans son atelier pour $y$ travailler avec acharnement ${ }^{33}$. Le résultat, qui est présenté à la Passedoit Gallery en janvier 1954, récolte un franc succès. À la fin de septembre, il élit domicile dans Greenwich Village, et prend connaissance des productions états-uniennes contemporaines au Whitney Annual of American Art. L'événement regroupe alors aussi bien des œuvres figuratives que de l'abstraction géométrique et de l'expressionnisme abstrait, Motherwell, Gottlieb, Baziotes, ainsi que Pollock qui, après sa période de dripping, effectue un retour à la figuration ${ }^{34}$. Borduas juge toutefois ces productions inférieures à celles des Européens, en raison de l'association qu'il établit avec le réalisme social qui, selon lui, demeure tributaire des nécessités externes ${ }^{35}:$ « [...] jamais dans ces exemples je n’ai ressenti la fortifiante impression de jamais vu, qui seul peut nous enrichir. Je considère toutes ces œuvres comme futiles, illusoires ou grossières $^{36}$." Quelques mois plus tard, il précise: «Dans l'ensemble, la peinture américaine souffre de son manque de cohésion; comme il n'y a pas de véritable esprit artistique, avec ses hérauts c'est la technique qui est l'élément primordial ${ }^{37}$. " Cette perception révèle alors son incompréhension des productions et ravive sa conviction que le renouveau viendra non pas du Sud, mais de l'autre côté de l'Atlantique $e^{38}$.

Pourtant, Borduas s'éveille bientôt au potentiel d'ouverture spatiale des œuvres de Pollock et de Kline qu'il situe «au-delà du

\footnotetext{
${ }^{33}$ Gagnon, Borduas and America let l'Amérique, p. 31. Voir également, au sujet du séjour new-yorkais, Thérèse Martin, « La structure spatiale de l'œuvre new-yorkaise de Paul-Émile Borduas (1953-1955)».

${ }^{34}$ Gagnon, Borduas and America let l'Amérique, p. 31.

${ }^{35} \mathrm{Ibid}$., p. 28.

${ }^{36} \mathrm{Ibid}$., p. 28. Musée d'art contemporain de Montréal. Notes biographiques (T130).

${ }^{37}$ Propos cités par Rodolphe de Repentigny (alias François Bourgogne), "Chez Borduas à New York", L'Autorité du peuple, 6 février 1954, p. 7.

${ }^{38}$ François-Marc Gagnon, «New York as Seen from Montreal by Paul-Emile Borduas and the Automatists », dans Guilbaut, dir., Reconstructing Modernism: Art in New York, Paris, and Montreal, p. 138.
} 
surréalisme ${ }^{39} "$. Avouant sa fascination pour les dimensions dynamique et matérielle de l'espace que - selon lui - Pollock assume totalement, il écrit: "L'accident, qu'il multiplie à l'infini, se montre alors capable d'exprimer à la fois la réalité physique et la réalité psychique sans le support de l'image ou de la géométrie euclidienne ${ }^{40}$." Or, Pollock nie l'accident ${ }^{41}$. L'intervention d'un "hasard contrôlé " lui permet de justifier l'équilibre entre l'ordre et le chaos, entre l'intervention spontanée du geste et les efforts conscients qu'il doit fournir pour uniformiser et équilibrer les composantes dans de vastes formats. Quant à la ligne, elle revêt un intérêt moins pour le contenu expressif qu' elle charrie ou ses conditions de réalisation par accidents successifs, que pour son potentiel spatial et dynamique. Au contact de cette nouvelle sensibilité, Borduas effectue un retour à l'aquarelle et à l'encre (après une première série en 1950-1951), dont la fluidité et la malléabilité lui permettent de vaquer à des expérimentations plus mécaniques et gestuelles ${ }^{42}$. Inspiré par les techniques du dripping et du splashing popularisées par Pollock ${ }^{43}$, mais sans toutefois faire

${ }^{39}$ Lettre de Borduas à Claude Gauvreau, New York, 25 septembre 1954. Musée d'art contemporain de Montréal, Fonds Borduas (T. 218). Écrits II, t. II, p. 651.

${ }^{40}$ Borduas, "Objectivation ultime et délirante", New York, 26 février 1955. Écrits I, p. 522-524.

${ }^{41}$ Jackson Pollock, "Entretien avec William Wright ", entrevue non diffusée. Script publié par F. V. O'Connor, dans Jackson Pollock, New York, MoMA, 1967, p. 79. Repris et traduit dans le catalogue Jackson Pollock, Paris, Centre GeorgesPompidou, 1982, $420 \mathrm{p}$.

${ }^{42}$ Thérèse Martin souligne également que ce médium offrait une qualité de séchage propice aux expérimentations diverses, et qu'elle s'est avérée également moins coûteuse et donc plus rentable que l'huile ( La structure spatiale de l'œuvre newyorkaise de Paul-Émile Borduas (1953-1955)», p. 42).

${ }^{43}$ François-Marc Gagnon, Paul-Émile Borduas, Montréal, Musée des beaux-arts de Montréal, 1988, p. 340; Martin, "La structure spatiale de l'œuvre new-yorkaise de Paul-Émile Borduas (1953-1955)", p. 59-60. L'expression dripping, tirée du verbe "drip " - laisser couler, tomber goutte à goutte, dégouliner - consiste non plus à appliquer le pigment sur la toile à l'aide du traditionnel pinceau, mais à laisser couler verticalement la peinture - désormais à l'émail, beaucoup plus fluide - sur la toile. Le résultat présente alors des entrelacs sinueux. Le splashing consiste, pour sa part, à " éclabousser » la surface, en projetant énergiquement le pigment, de manière à générer des segments plus saccadés. Le lecteur pourra consulter à ce sujet le site: http://www.tate.org.uk/liverpool/ima/rm4/resources/. 
appel au all-over composition, Borduas réalise notamment Le Chant du guerrier, Gerbes légères, Bombardement délicat, Les Baguettes joyeuses, Figure cabalistique (1954) ${ }^{44}$. Contrairement au dripping, la forme prend toutefois le dessus sur le mouvement, reléguant ainsi son travail à une étape antérieure de la tradition tachiste. L'articulation entre l'espace et le mouvement, entre la forme et la vie, qui se trouve synthétisée, chez Pollock, dans le concept de "potentiel ", n'est pas assimilée à ce stade chez Borduas, qui ne conçoit l'accident que comme un moyen d'organiser le monde à partir d'un éclatement de sa structure et de ses formes.

Borduas réalise ensuite une série, comprenant notamment Blanches figures, Trouées blanches, Échancrure, L'Aigle à la blanche famille (1954), inspirée cette fois de l'aspect calligraphique des œuvres de Franz Kline, de leur disposition à lier l'espace et la forme ${ }^{45}$. Thérèse Martin a souligné que, chez Kline, la tension entre le trait noir et l'espace blanc se trouve maximisée, jusqu'à intervertir leur statut (la forme se veut espace et inversement), alors que chez Borduas, cette tension entre les masses sombres et claires tend plutôt à se maintenir et à s'équilibrer, créant une "lutte vibratoire ${ }^{46}$. Les œuvres Frais

${ }^{44}$ Plusieurs des œuvres mentionnées peuvent être consultées dans le catalogue de Gagnon, Paul-Émile Borduas. Le catalogue raisonné en ligne (http://borduas. concordia.ca/catalogueRaisonne/Recherche.php), présenté par Gagnon, n’offre pas encore, à ce jour d'illustrations des œuvres.

${ }^{45}$ François-Marc Gagnon, Paul-Émile Borduas (1905-1960). Biographie critique et analyse de l'auvre, Montréal, Fides, 1978, p. 362. À ce titre, Gagnon suggère, sous toute réserve, que Borduas aurait vu les œuvres de Kline à l'exposition Younger American Painters présentée au Guggenheim Museum en mai 1954 (Borduas and America let l'Amérique, p. 36). Thérèse Martin a rapporté le témoignage de la peintre Yvonne Thomas, qui avait côtoyé Borduas lors de son séjour, et attesté que ce dernier a participé à des discussions au Club où se réunissaient plusieurs artistes de l'École de New York. Kline et Borduas seraient alors entrés en relation. («La structure spatiale de l'œuvre new-yorkaise de Paul-Émile Borduas (19531955) ", p. 55, note 16). Voir également Écrits II, t. II, p. 651, note 239. Pour un aperçu des œuvres de Kline, consulter: http://www.beatmuseum.org/kline/ newyork.html.

${ }^{46}$ Martin, "La structure spatiale de l'œuvre new-yorkaise de Paul-Émile Borduas (1953-1955)», p. 65. Rodolphe de Repentigny soulignait à propos de l'œuvre Blanches figures: « [...] les espaces blancs sont traités avec autant d'attention que 
jardin, L'on a trop chassé, Coups d'ailes à Bonaventure (1954), Forêt métallique ${ }^{47}$ ou Ardente (1955) renferment toutefois des composantes qui, davantage libérées de leur statut représentationnel et soutenues par de généreux empâtements, tendent à lier forme et matière. Malgré cet apport, les œuvres évoquent par moments l'espace réel, renforcé par l'intervention de l'accident qui produit des entrechoquements entre les motifs, mais également par les titres, comme en font foi les taches rouges qui ponctuent la surface de L'on a trop chassé, ou encore les facettes grisâtres juxtaposées aux taches blanches dans Coups d'ailes à Bonaventure. L'uniformisation progressive de l'organisation spatiale a toutefois pour effet, paradoxalement, de ramener les éléments connus à des effets purement sensitifs, comme l'exprime Borduas: "La sensation de la profondeur est devenue immédiate et infinie. Complète objectivation qui élimine toute l'illusion de la perspective aérienne ${ }^{48}$.» " Mon seul jugement valable en face de mon travail est le vertige d'une reconnaissance essentiellement émotive provoquée par la sensation d'une synthèse généreuse ${ }^{49}$." Cette nouvelle conception de l'espace en termes de sensation et d'expériences génériques éloigne désormais l'œuvre de la "nécessité interne » des surréalistes.

\section{Entre l'espace réel et l'espace pictural}

Malgré les nombreux virages et détours inattendus qui caractérisent la production borduasienne des années $1950^{50}$, on remarque que la dimension matérielle de la matière picturale (painterliness) tend à s'affirmer progressivement pour atteindre une certaine "pureté

les [...] taches noires" ("Trois peintres: Borduas, Raymond et Wilson ", La Presse, Montréal, 29 octobre 1955, p. 1-2, 75).

${ }^{47}$ Gagnon, Paul-Émile Borduas, p. 387.

${ }^{48}$ Lettre de Borduas à Noël Lajoie, New York, 11 février 1955. Fonds privé. Écrits II, t. II, p. 717.

${ }^{49}$ Borduas, Paris, 10 avril 1956. Réponse à un questionnaire envoyé par Jean-René Ostiguy. L'auteur souligne.

${ }^{50}$ Martin, «La structure spatiale de l'œuvre new-yorkaise de Paul-Émile Borduas (1953-1955)», p. 28. 
plastique ${ }^{51}$ ", sans abandonner complètement les références au réel. Malgré une uniformisation progressive de la surface, la dichotomie figure/fond se trouve partiellement conservée, comme le démontrent Figures aux oiseaux et Danse sur le glacier (1953). Les éléments réduits à des facettes claires et sombres ponctuées d'éclats colorés sont peu à peu libérés de leur charge expressive, et deviennent de purs signes picturaux, qui gagnent en dynamisme, comme l'atteste par son titre Les signes senvolent (1953) présenté à l'exposition solo En route! (galerie Agnès Lefort, octobre 1954) ${ }^{52}$. Les facettes colorées, qui sont peu à peu absorbées par la dominance des zones lumineuses, ont pour effet de diluer peu à peu les détails et les ombres, et de minimiser par conséquent les contrastes et l'effet creusant. Dans une entrevue qu'il accordera en 1957, Borduas explique rétrospectivement les transformations provoquées par l'abandon de la couleur et le renforcement de la structure dialectique des éléments:

Cette perte de la couleur s'est faite très graduellement pour rejoindre une plus grande efficacité, une plus grande visibilité, une plus grande objectivité de contraste [...] il n'y a plus de perspectives: ni aérienne ni linéaire, mais quand même, toujours, la troisième dimension, mais une troisième dimension qui est exprimée sans le recours de toute une série de plans. Dans les derniers tableaux, [...] la couleur jouait justement ce rôle d'intermédiaire d'un plan à l'autre. Et comme les intermédiaires ont sauté, la couleur a sauté avec... ${ }^{53}$

Borduas accorde désormais une plus grande importance à la matière et à la structure, comme le témoigne Les Boucliers enchantés (1953) dont les touches étalées plus en espace qu'en volume décrivent un élan vertical ${ }^{54}$. Cette structure dynamique, qui bloque toute percée

\footnotetext{
${ }^{51}$ Rodolphe de Repentigny avait souligné ce tournant, dans un article consacré à la première exposition de Borduas à New York: "Exposition Borduas à la Galerie Passedoit ", L'Autorité du peuple, Montréal, 6 février 1954, p. 7.

${ }^{52}$ Gagnon, Paul-Émile Borduas, p. 318.

${ }^{53}$ Entrevue accordée à Judith Jasmin, émission Carrefour, Radio-Canada, 1957. Retranscription dans Paul-Émile Borduas, Refus global et autres écrits, p. 291-293.

${ }^{54}$ Gagnon, Paul-Émile Borduas, p. 332.
} 
du regard, est d'ailleurs suggérée par le titre. Si de nombreux titres renvoient à des éléments de la nature ${ }^{55}$ (rochers, oiseaux, branchages, glaces, plans d'eau, etc.) et ramènent le spectateur à une expérience connue, celle-ci évoque non pas directement un motif, mais plutôt son mouvement dans l'espace (envolée, avancée, danse, flottaison), une condition de stagnation ou encore le mouvement qui suit cette immobilité (dégel). Des titres comme Pulsation (1955) et Expansion rayonnante (1956) (figure 1) charrient d'ailleurs littéralement la dimension sensitive de l'élan, la dynamique dualiste de l'action d'un corps succédant à son immobilité. Ce mouvement élémentaire surgi d'un épicentre synthétise étrangement la situation de l'artiste face à son environnement. Dans une lettre qu'il rédige au tout début de janvier 1953 à Saint-Hilaire, à l'attention de sa fille Jeanine, alors que son départ pour les États-Unis est retardé par les complications administratives pour l'obtention de son visa, Borduas exprime, entre deux faits divers, cet état dualiste: "J'ai l'impression d'être au seuil d'une certaine fureur [...] La glace est prise. C'est la blanche immobilité pour jusqu'au printemps ${ }^{56}$." Un an plus tard, alors qu'il est installé à New York, le même sentiment d'entre-deux ressurgit: "J'ai l'impression d'être entre mon passé et l'avenir: plus près cependant de l'avenir - dans une action où de nouveaux espoirs se précisent chaque jour davantage $[\ldots]^{57}$. " De sa peinture, Borduas tient le même discours: "J'ai l'impression qu'elle a fait un bond, comme un bouchon qu'on aurait longtemps retenu sous l'eau et qui se sent libre tout à coup. Des œuvres sombres et sévères du passé je vais à l'éblouissement, au vertige prochain, j'espère, de la lumière, de

${ }^{55}$ Selon Nathalie Clerk, les productions des années 1940 conservaient également un "attachement à une thématique de la nature ou de l'homme" (La peinture de Borduas de 1941 à 1948: en quoi reste-t-elle encore attachée à la figuration?, p. 54).

${ }^{56}$ Lettre de Borduas à sa fille Jeanine, 4 janvier 1953. Fonds privé. Écrits II, t. II, p. 501 .

${ }^{57}$ Lettre de Borduas à Rodolphe de Repentigny, New York, 12 janvier 1954. Publiée dans l'Autorité du peuple, le 6 février 1954, p. 7. et dans la Presse, le 27 février 1960, p. 33. Ecrits II, t. II, p. 569. 
l'espace, de la matière ${ }^{58}$ ! Cette transition est d'ailleurs soulignée par Jean Éthier-Blais: «Les toiles peu à peu se libèrent de tout sauf du mouvement et de la lumière. [...] La matière-lumière passe entièrement au service de la forme-mouvement ${ }^{59}$. " Cette dynamique traverse d'ailleurs des œuvres comme Figures aux oiseaux (1953), La Blanche envolée (1954), Sea Gull (1956) ${ }^{60}$, qui évoquent un mouvement aérien ou des volatiles qui se déploient dans un espace ouvert, tandis que L'Étang recouvert de givre, Miroir de givre, Jardin sous la neige (1954), Forêt métallique (1955), Le Chant de la pierre et Le Dégel $(1956)^{61}$, réferent à la surface uniformisée et infinie des paysages enneigés, des glaces polaires, ou à une structure tectonique comparable au mouvement de débâcle. Si certains critiques nient toute référence au réel, d'autres soulignent l'évocation d'une expérience nordique, non seulement par la clarté lumineuse des motifs, mais surtout par l'uniformité spatiale et une certaine " turbulence » des composantes ${ }^{62}$. À ce titre, les références à l'espace réel, à une perspective icarienne ou aérienne ${ }^{63}$, renvoient à l'expérience sensitive ou conceptualisée d'un mouvement d'ouverture et d'un décloisonnement des frontières à la fois géographique et psychologique.

La récurrence de la condition hivernale présente un autre registre de sensations qui caractérise l'expérience des populations nordiques. Les spécialistes de la littérature et des mythologies canadiennes

\footnotetext{
${ }^{58}$ Lettre de Borduas à Guy Viau, New York, 4 mars 1954. Musée du Québec. Fonds Guy Viau. Écrits II, t. II, p. 572.

${ }^{59}$ Jean Éthier-Blais, «Épilogue et méditation», Liberté, vol. 4, n 22 (avril 1962), p. 257.

${ }^{60}$ Gagnon, Paul-Émile Borduas, p. 339, 351. Une reproduction de Sea Gull est également disponible à l'adresse suivante: http://cybermuse.gallery.ca/cybermuse/ enthusiast/thirties/artwork_f.jsp?mkey=3989.

${ }^{61}$ Gagnon, Paul-Émile Borduas, p. 353, 386, 447.

${ }^{62}$ Carlyle Burrows, "Art: Native and Foreign Features", The New York Herald Tribune, 24 mars 1957, p. 11.

${ }^{63}$ Fernande Saint-Martin soutient que «Borduas posa les constantes du paysage où les éléments sont pour ainsi dire fusionnés par le lien atmosphérique et il élabora une dialectique si particulière de la perspective aérienne " (Les structures de l'espace pictural, Montréal, HMH, 1968, p. 133).
} 
s'entendent pour affirmer que la nature et le climat canadiens auraient sur ses habitants l'effet contemplatif d'élever l'âme au grandiose, tout en s'imposant du même coup comme une force essentiellement hostile, qui écrase et tend à réduire à néant les efforts d'adaptation et de survie ${ }^{64}$. Cette condition qui implique un rapport dualiste à l'environnement rejoindrait l'expérience de Borduas, non pas dans sa dimension proprement physique ou climatique, mais plus précisément dans les instabilités émotionnelles de l'artiste, peuplées d'emballements et de vertiges. Provoquées par ses contraintes d'exil, ces instabilités se trouveraient synthétisées dans la présence de plus en plus affirmée des blancs et des noirs, de la lumière et de l'ombre, de l'ouverture et du renoncement ${ }^{65}$. L'apaisement que procure à l'artiste son éloignement des épuisantes frustrations du milieu québécois, ainsi que les premières reconnaissances qu'il récolte du milieu new-yorkais, ont toutefois pour effet de neutraliser en partie ces va-et-vient. Rodolphe de Repentigny remarque d'ailleurs, à propos des œuvres, que, malgré les tensions qui l'animent, la surface uniformisée engendre un sentiment de liberté et de "sérénité", comme l'a résumé Gagnon : "[...] le traitement all over a un effet apaisant sur le spectateur. Comme il ne lui impose aucun point focal, il invite au vagabondage du regard, à l'errance insouciante d'un point à un autre de la surface, et il engendre une sorte d'euphorie et de paix ${ }^{66}$. " Malgré l'arbitraire de sa réception, ce

${ }^{64}$ Voir notamment Margaret Atwood, Essai sur la littérature canadienne, traduit de l'anglais par Hélène Filion, Montréal, Boréal, 1987 [1972], p. 47; Northrop Frye, The Bush Garden: Essays on the Canadian Imagination, Toronto, Anansi, 1971, 256 p.; Douglas Gordon Jones, Buttlerfly on Rock. A Study of Themes and Images in Canadian Literature, Toronto et Buffalo, University of Toronto Press, 1970, 197 p.; Bruce Littlejohn et Jon Pearce, Marked by the Wild. An Anthology of Literature Shaped by the Canadian Wilderness, Toronto, McClelland and Stewart, 1973, 287 p.; Gaile McGregor, Wacousta Syndrome: Explorations in the Canadian Landscape, Toronto, University of Toronto Press, 1985, 473 p.

${ }^{65}$ Françoise Cloutier-Cournoyer, Structure de l'espace pictural dans l'œuvre parisienne de Paul-Émile Borduas (1955-1960), Mémoire de maîtrise (histoire de l'art), Université de Montréal, 1974, p. 42.

${ }^{66}$ Gagnon, Paul-Emile Borduas, p. 319. Rodolphe de Repentigny, "Incessante évolution de Borduas", La Presse, Montréal, 16 octobre 1954, p. 74. 


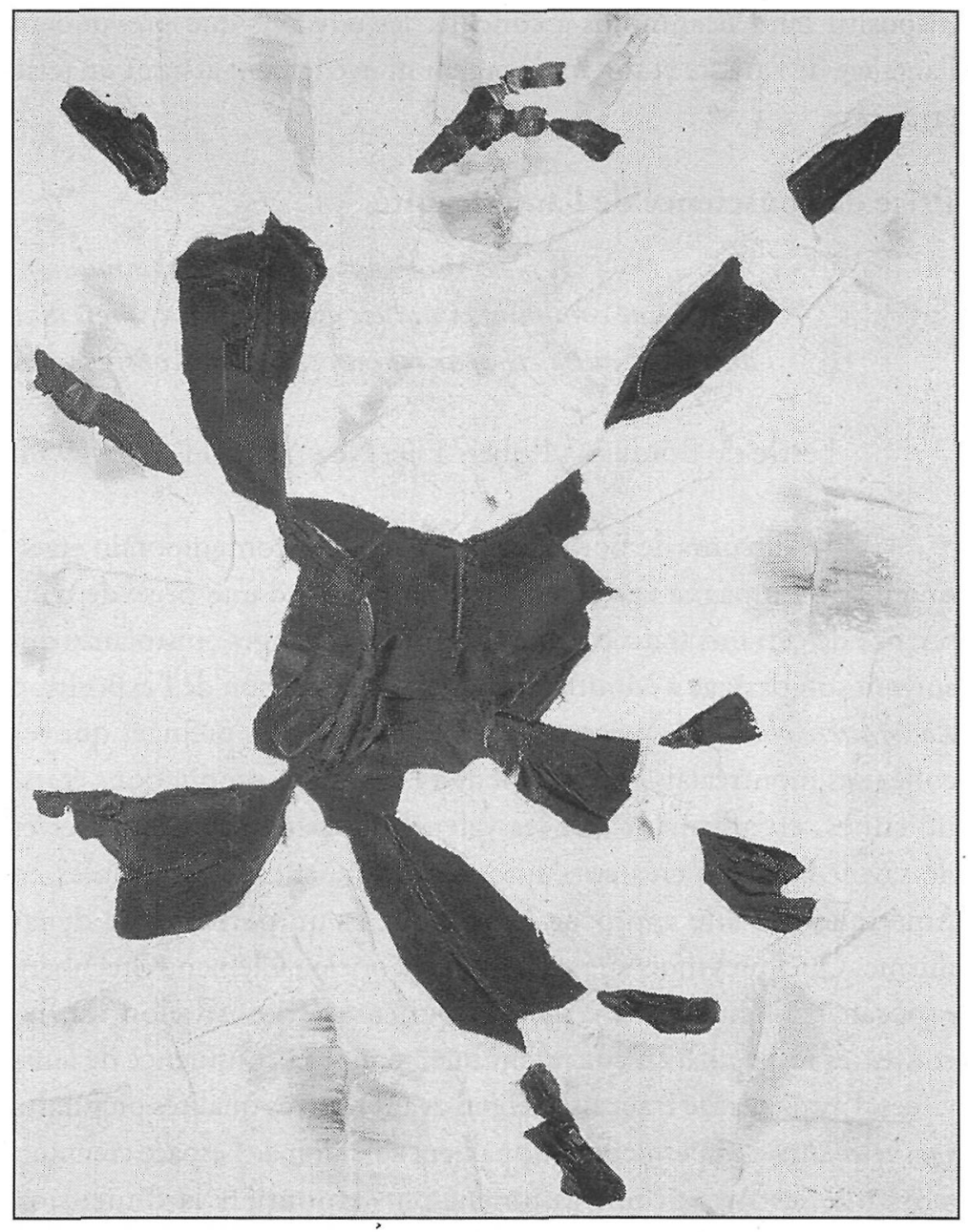

Figure 1 - Paul-Émile Borduas, Expansion rayonnante, 1956; huile sur toile. Musée des beaux-arts de Montréal (Don du Dr et de $\mathrm{M}^{\mathrm{me}}$ Max Stern; $\mathrm{n}^{\circ}$ 1978.22; photo: Marilyn Aitken, MBAM). 
dispositif tend néanmoins à concilier les tensions que provoquent l'ancrage de l'artiste et son mouvement nouvellement assumé en terre étrangère.

\section{Prise de conscience de l'américanité}

À New York nous avons cette communion, une communion qui est assez abstraite, mais avec l'univers et qui a été pour moi un stimulant considérable.

Lettre de Borduas à Robert Élie, New York, 11 mars 1954.

Le discours de Borduas à l'égard de l'art contemporain étatsunien s'accompagne également, par moments, d'une prise de conscience de certains traits culturels. Dans les échanges épistolaires qui suivent son passage à Montréal, en 1954, à l'occasion de l'exposition La Matière chante, le peintre s'efforce de réviser les préjugés que ses collègues montréalais entretiennent à l'égard des prodùctions étatsuniennes, en affirmant que les valeurs d'expérience, de métier et de savoir-faire des créateurs européens, auraient été remplacées en Amérique par une approche heuristique et un potentiel de dynamisme. Quelques mois auparavant, le théoricien Clement Greenberg exposait d'ailleurs les divergences qui caractérisent, selon lui, les contextes respectifs, en concluant que, malgré la confluence de leurs visées, l'avant-garde française défend avant tout les qualités picturales (paint quality) qui permettent de pallier l'abandon de l'espace creusant, alors que les Américains assument plus volontiers la dimension ontologique de la matière et de l'espace, la réalité d'un vide à remplir : "The American version is characterized, in failure as well as in success, by a fresher, opener, more immediate surface. Whether it is enamel reflecting light, or thinned paint soaked into unsized and unprimed canvas, the surface breathes ${ }^{67}$. "Si le nom de Greenberg et ses théori-

${ }^{67}$ Clement Greenberg, "Symposium: Is the French avant-garde overrated? ", Art Digest, vol. 27, nº 20 (septembre 1953), p. 12. 
sations du travail des avant-gardes ne circulent pas encore largement à l'époque, Borduas reconnaît d'instinct que l'orientation artistique américaine s'inscrit non seulement en continuité avec la tradition européenne, mais qu'elle en renouvelle le développement: Il prend également conscience de la brèche qu'elle opère dans les schèmes européens. À la veille de son départ pour Paris, en septembre 1955, il explique alors à un journaliste du Montreal Star ce qui, selon lui, distingue les peintures européenne et américaine:

Yet the feeling of the North American painter is entirely different from the European. This feeling is still very loose and indefinable; it is an emotional, not an intellectual one. Our sensibility tolife is perhaps a bit "rough" and not so sophisticated as the old world's, but it is this very naivveté that gives us youthfulness and freshness ${ }^{68}$.

Selon Gagnon, Borduas réalise que, chez ses homologues étatsuniens, les dimensions pragmatique, empirique, et la réalité concrète l'emportent sur l'intériorité et la pensée, contrairement aux avantgardes européenne et montréalaise qui misent plus spécifiquement sur les valeurs de l'esprit et les nécessités internes ${ }^{69}$. Il entrevoit alors la possibilité de réconcilier ces réalités: "Les plus profondes résonnances humaines, de notre époque, viennent de cette intimité, EspritMatière. Équivalence totale, où l'esprit n'apparaît que l'accident organique de cette matière; ou la matière l'accident organique de l'esprit ${ }^{70}$. " $\mathrm{La}$ dichotomie traditionnelle entre l'esprit associé à la réflexion européenne, et la matière attribuée à une Amérique longtemps perçue comme matérialiste et mercantile, se voit ainsi transcendée au profit d'une

${ }^{68}$ Paul-Émile Borduas, "New York. Talk with an artist», The Montreal Star, 9 juin 1955.

${ }^{69}$ Gagnon, "La première expérience de la peinture américaine abstraite (les années 1950)", p. 277. Dans une lettre à Fernand Leduc, Borduas précise: «Bien des fois je l'ai dit, ESPRIT et MATIÈRE sont inséparables; si l'un est éternel l'autre l'est aussi [...] ESPRIT?... Qualité de la matière; ou, la MATIÈRE serait la qualité de l'esprit " (Lettre à Fernand Leduc, Saint-Hilaire, $1^{\text {er }}$ novembre 1949. Fonds privé. Écrits II, t. I, p. 347-350). À propos de l'avant-garde états-unienne, Margit Rowell soulignait que "La nouvelle peinture précède la pensée; elle ne peut être saisie par l'esprit qu'ultérieurement" (La peinture, le geste et l'action, p. 30).

${ }^{70}$ Lettre de Borduas à Claude Gauvreau, New York, 25 mars 1954. Fonds privé. Écrits II, t. II, p. 578. 
tension créatrice. L'ancien professeur, qui a longtemps souffert d'un complexe d'infériorité auprès de ses pairs en raison de ses origines modestes et d'une soi-disant carence d'instruction, aurait probablement trouvé un réconfort dans ce contexte où le clivage entre culture savante et culture populaire est atténué, et où les valeurs de l'expérience et de l'expérimentation sont reconnues. Pourtant, son manque de recul face à l'importance que prend alors l'avant-garde américaine sur l'échiquier de l'avenir de l'art le pousse à regagner Paris. Sa difficulté à maîtriser l'anglais a sans doute participé également à sa décision ${ }^{71}$. Fortement marqués par le pôle d'attraction que constitue encore la capitale française, en dépit de ses commotions récentes, la majorité des artistes du Québec ressentent d'ailleurs le besoin de compléter le processus de rattrapage culturel et de neutraliser certains conflits séculaires issus de la dynamique coloniale. De son premier voyage en France, Borduas conserve également l'image d'une prestigieuse terre de culture et d'histoire, ainsi que le souvenir de découvertes trop brusquement interrompues par la crise. Il continue du même coup d'y voir un ultime espoir de consécration : «Seul Paris peut faire vivre un peintre et sa peinture ${ }^{72}$. En réalité, il y récoltera plus de contrariétés qu'à New York, comme nous le verrons ${ }^{73}$.

La proximité que les artistes états-uniens assument avec l'environnement immédiat, et qui se trouve exprimée, dans les œuvres, par une interpénétration de l'espace réel et de l'espace du tableau, et par l'assomption de la matérialité du pigment et du support, rejoint également certains préceptes primitivistes selon lesquels les artistes se croient plus aisément disposés à effectuer un retour aux sources,

${ }^{71}$ Jean-Pierre Denis, "Borduas hors frontière. Le One Big world», dans Guy Bellavance, dir., Monde et réseaux de l'art. Diffusion, migration et cosmopolitisme en art contemporain, Montréal, Liber, 2000, p. 39.

${ }^{72}$ Lettre de Borduas à Marcelle Ferron, New York, 8 février 1954. Fonds privé. Écrits II, t. II, p. 571.

${ }^{73}$ Gagnòn, Borduas and America let l'Amérique, p. 43. Borduas avoue un an après son arrivée: «Il semble qu'il eût été préférable de ne pas quitter New York" (Lettre à Renée Borduas, Paris, décembre 1956, cachet postal du 15 décembre. Fonds privé. Écrits II, t. II, p. 877. 
à une dimension pré-culturelle aussi bien intime qu'historique, compte tenu de leur relatif isolement du fardeau culturel et historique européen ${ }^{74}$. L'écrivain Amin Maalouf a récemment montré ce paradoxe: «Quand la modernité porte la marque de l'“Autre”, il n'est pas surprenant de voir certaines personnes brandir les symboles de l'archaïsme pour affirmer leur différence ${ }^{75}$." En 1957, Borduas émet un nouveau bilan de la situation de la peinture, en notant un changement significatif dans la conception américaine de l'espace:

Depuis cinq ou six ans, à New York, un mouvement vers un changement d'échelle s'est opéré: les tableaux sont devenus de plus en plus grands, de plus en plus simples, en comportant de moins en moins d'éléments. On a l'impression que la peinture de New York va vers des généralisations possibles, tandis que l'impression que nous avons à Paris est, au contraire, que ça va vers un art de plus en plus intime, de plus en plus personnel, de plus en plus psychique ${ }^{76}$.

L'artiste laisse alors entendre que l'élargissement de l'espace et la simplification de son contenu seraient justifiés par l'affirmation d'un point de vue générique, «impersonnel ", sur une condition humaine élargie, qui assume ses tiraillements, ses contradictions et son dualisme, alors que l'échelle plus restreinte de la peinture européenne serviraient l'expression d'un contenu davantage individuel, intime, spécifique. Au-delà des idéaux universalistes que l'Amérique incarne dans le climat d'après-guerre, Borduas entretient d'ailleurs, dès 1954, dans la conscience ce qu'il appelle un «sentiment nord-américain", une vision du mythe du renouveau perceptible à travers les valeurs d'action:

Ce sentiment venait d'une certaine verdeur retrouvée dans la musique américaine et un peu partout dans les gestes: verdeur lyrique et virginale. Ce sentiment ne m’a plus quitté depuis [...]

\footnotetext{
${ }^{74}$ Rowell, La peinture, le geste, l'action.

${ }^{75}$ Amin Maalouf, Les identités meurtrières, Paris, Grasset, 1998, p. 99.

${ }^{76}$ Entrevue de Paul-Émile Borduas par Judith Jasmin, Paris, le 2 mai 1957, Télévision française, Émission Carrefour, Archives en ligne de Radio-Canada. Transcription dans Borduas, Refus global et autres écrits, p. 292-293.
} 
Certes il est épatant qu'en Amérique l'on soit si ardent pour les aventures sportives. Nous sommes ainsi en pleine magie du mouvement et Dieu sait si les corps m'intéressent. Il est bon de sentir un enthousiasme collectif quelque part ${ }^{77}$.

Dans ce contexte, l'expression synthétique du mythe, qui demeure universellement partageable et renouvelable, permet de réitérer les enjeux existentiels et de dénouer ses tensions collectives, grâce à une dynamique d'accomplissement ${ }^{78}$. C'est toutefois au cours de sa période parisienne que sera éprouvé, chez Borduas, ce sentiment qui donnera alors naissance à une nouvelle perspective spatiale:

Ma peinture a fait un bond simplificateur considérable. Elle est devenue de larges taches noires comportant leur propre lumière par la modulation de la matière sur un fond blanc également modulé dans la pâte et par des gris qui s'y noient. Contraste limite, objectivation limite, et je pense aussi, distance limite entre les idées si claires, si primaires qui les suscitent et l'émoi incompréhensible qui en résulte ${ }^{79}$.

Au cours de l'été 1956, Borduas entame un long périple en solitaire à travers l'Italie, la Sicile, puis l'Espagne et la Grèce. Cet arrachement à sa réclusion parisienne a alors un effet bénéfique. À bord d'une voiture décapotable, il parcourt des milliers de kilomètres pour découvrir non seulement les sources de l'art occidental, mais aussi une nouvelle conception de l'espace. À son retour, il déclare: "Je me sens [...] gourmand, gourmand. Je voudrais meubler tout l'Espace d'un seul plan ${ }^{80 ! " ~}$

Un long voyage en Sicile a eu le don d'accentuer la rupture avec l'échelle européenne au profit d'une certaine grandeur grecque et romaine qui n'existe plus à partir du christianisme. Grandeur morale. Prise de possession de l'espace à l'échelle du paysage. C'est déjà le germe des meilleures réalisations nord-américaines.

77 Lettre à Gilles Corbeil publiée sous le titre «Paul-É. Borduas nous écrit au sujet d'Ozias Leduc", Art et Pensées, juillet-août 1954, p. 177-179.

${ }^{78}$ À ce sujet, voir Andrès et Bouchard, dir., Mythes et sociétés des Amériques.

${ }^{79}$ Lettre de Borduas à Michel Camus, Paris, 5 août 1956. Fonds privé. Écrits II, t. II, p. 853-854.

${ }^{80}$ Lettre de Borduas à Bernard A. Bernard, Paris, 11 novembre 1956. L'artiste souligne. Fonds privé. Écrits II, t. II, p. 869. 
Un monde merveilleux se construit qui va tout faire sauter de l'exécrable isolement chrétien: isolement de l'âme dans le refus de l'univers ${ }^{81}$.

En établissant un parallèle entre l'héritage mythologique de l'Antiquité grecque et latine et celui de l'Amérique de la frontière, Borduas saisit la manière dont l'action signifiante de l'individu dans l'espace confere au territoire une dimension mythique. À travers ce périple, le retour aux sources qu'il avait longtemps envisagé prend un sens nouveau, direct, pragmatique. Marqué par cette vision, le peintre réitère ses convictions: «ma peinture file vers un autre monde plus impersonnel, plus général. Fini, pour moi, les petites bêtises sympathiques. C'est tout l'univers que j'ai besoin de saisir à la grecque, à la romaine, à l'américaine ${ }^{82} ! . . . »$

\section{Entre l'arrachement et l'enracinement}

Il faut être profondément enraciné quelque part: les êtres et les esprits flottants sont d'un pauvre intérêt.

Lettre de Borduas à Claude Gauvreau, New York, 16 sept. 1954

Projetée à une telle échelle, cette condition ne s'avère toutefois vivable que si un point d'ancrage modère le vertige qu' elle provoque ${ }^{83}$. Borduas se surprend d'ailleurs, parallèlement, à ressentir une nostalgie occasionnelle à l'égard de New York ou de Saint-Hilaire. En 1956, à la suite du décès de sa mère et d'Ozias Leduc, il avoue: «J'envie les destins bien enracinés, que rien de dérange, et qui s'écoulent tout

\footnotetext{
${ }^{81}$ Lettre de Borduas à Noël Lajoie, Paris, 29 nov. 1956. L'artiste souligne. Fonds privé. Écrits II, t. II, p. 875 .

${ }^{82}$ Lettre de Borduas à Claude Gauvreau, Paris, 22 décembre 1956. L'artiste souligne. Fonds privé. Écrits II, t. II, p. 889.

${ }^{83}$ Sur la nécessité d'établir des repères dans les représentations de l'infini, voir Anne Cauquelin, L'invention du paysage, Paris, Quadrige/PUF, 2000, p. 108-109, 124-125.
} 
doucement sur place ${ }^{84}$. " Au terme de chacun de ses périples à travers l'Europe, il ressent toutefois une répugnance à l'idée de retrouver le contexte parisien envers lequel il ne ressent pas de réelles affinités et qui entre d'ailleurs en conflit avec son reniement du mythe de la parenté culturelle avec la France. En conséquence de cette rupture, Borduas accorde au confort de son atelier, à son efficacité à distribuer la meilleure lumière, une importance centrale, obstinée, dont il se plaint lui-même de l'ampleur à travers sa correspondance ${ }^{85}$. Cette hantise révèle alors sa difficulté à s'adapter aux nouvelles données sensitives de la vie parisienne. Gilles Lapointe note à ce sujet: "Véritable représentation du monde intérieur de l'artiste, l'atelier prend valeur de symbole, recrée le microcosme, représente le cordon ombilical qui relie le peintre à son passé et à son lieu d'origine ${ }^{86}$." Sur le plan esthétique, Borduas se tourne du même coup vers la dimension matérielle de la peinture, qui assure une inscription dans un univers familier, un espace significatif, en accord avec les valeurs d'authenticité et d'intégrité qu'il n'a jamais reniées. Les épais empâtements qui caractérisent les œuvres semblent alors jouer un rôle d'ancrage dans le réel ${ }^{87}$, sans toutefois évoquer le terroir ou contrevenir à l'idéal d'universalisme, comme l'exprime l'artiste, qui

${ }^{84}$ Lettre de Borduas à Margot Bernard, Paris, 30 avril 1956. Fonds privé. Écrits II, t. II, p. 835. Plus tard, en 1958, il déclarera encore: «si je ne crève pas en route, un jour je retournerai au pays, m'achèterai un petit coin au bord d'un lac, y construirai une cabane et vivrai - jusqu'à ce que mort s'ensuive - de pêche et de chasse!..." Lettre de Borduas à Jeanne Brisebois, Paris, 7 février 1958. Fonds privé. Ecrits II, t. II, p. 972.

${ }_{85}$ Â Paris, Borduas aurait passé un temps considérable à chercher un atelier et à assurer son confort, en se plaignant de l'exigüité, du manque de commodité et du froid.

${ }^{86}$ Gilles Lapointe, "Borduas, entre New York et Paris ", dans Gauvin, Les automatistes à Paris, actes d'un colloque, p. 27. L'auteur s'appuie notamment sur le témoignage de Robert Élie qui affirmait que sa visite de l'atelier de Borduas, à New York, lui avait laissé l'impression qu'il y avait recréé celui de Saint-Hilaire ("Témoignage sur Borduas ", dans Euvres, Montréal, Hurtubise HMH, 1979, p. 605).

${ }^{87}$ Voir Marcel Saint-Pierre, «La pensée plastique de Borduas ", Paul-Émile Borduas, Montréal, Musée d'art contemporain de Montréal, Les 400 coups, 1998, p. 40. 
va même jusqu'à confondre la matière picturale aux réalités territoriale et tellurique: "Au fond, l'élément du monde qui demeure le plus permanent, le seul peut-être, c'est la peinture, la peinture physique, la matière, la pâte. C'est là mon sol natal, c'est ma terre. Sans elle je suis déracinée. Avec elle, que je sois à Paris ou ailleurs, peu importe - je suis chez moi ${ }^{88}$." C'est ainsi que l'artiste a transposé dans la matière picturale les idéaux de plénitude individuelle qu'il n'a pas été en mesure de concrétiser au sein de sa propre communauté. Comme si, en abandonnant ses anciens repères nationaux et régionaux, la matière s'était substituée à ses aspirations, et du même coup, aux intermédiaires rhétoriques et à la charge expressive qu'elle révélait auparavant.

\section{Redéfinir les attaches culturelles}

La réévaluation des schèmes d'appartenance donnera naissance, chez Borduas, à une réflexion sur la redistribution éventuelle des espaces culturels nord-américains, et sur la place que le Québec occupera sur ce nouvel échiquier. En 1958, le peintre explique à Claude Gauvreau le rôle de l'avant-garde américaine dans le renouvellement de l'espace pictural, lequel s'accorde avec l'idée de renaissance culturelle forgée par la mythologie américaine:

Une équipe nombreuse de peintres exceptionnels a donné au Monde les deux éléments indispensables à l'élaboration d'un futur prestigieux : la libération et l'accident "objectif " impersonnel (contrairement à l'accident psychique "personnel " de Wols) et un nouveau concept de l'espace. Pour une fois, de toute l'histoire de l'art, l'appréhension méditerranéenne (visuelle) du Monde éclate. Et pour une fois tout signe peut rejoindre ses inconnues. Quelle aventure et combien débordante! Un vertigineux début

\footnotetext{
${ }^{88}$ Jean Éthier-Blais, "Conversation rue Rousselet", Vie des arts, vol. 5, n 19 (été 1960). Écrits I, p. 674. Johanne Lamoureux a également relevé cette adéquation en parlant de "greffe territoriale ", dans le cadre d'une table ronde radiophonique, Radio-Canada, $1^{\text {er }}$ avril 1997. Propos rediffusés dans la $15^{\circ}$ émission de la série "Du Refus global au village global ", Radio-Canada MF, 22 mai 1998.
} 
de synthèse (des races d'abord - des éléments de tous les peuples de la Terre réunis en Amérique épousent spontanément un même enthousiasme - de la connaissance ensuite - de toute l'expérience historique) s'élabore dans une étendue vierge à l'échelle cosmique laissant infiniment loin en arrière les exemples du passé. Comment, devant ces réalisations, attacher encore de l'importance à nos petites misères entachées d'archaïsme quand en plus, tragiquement, nous poursuivons le même destin? Et voilà le deuxième article de ma foi : L’Amérique du Nord poussera suffisamment la synthèse universelle pour rayonner sur la Terre entière ou nous n'existerons pas; ni elle, ni nous. C'est l'enjeu capital de l'histoire ${ }^{89}$.

De l'avis du peintre, cette synthèse constitue du même coup un moyen par lequel la communauté canadienne-française serait en mesure de résoudre certains conflits séculaires, comme il le confesse cette fois à David A. Gibbs:

Il est étrange [...] que l'on nous prête deux natures s'opposant : l'une canadienne ou américaine, l'autre européenne. Il est douloureux d'entendre que nous ayons à opter pour ou contre l'Amérique $\mathrm{du}$ Nord quand nous sommes de vieux Canadiens d'un seul jet. La trahison ne peut certes pas être une solution ni aimable, ni généralisable. Non! Si nous n'arrivons pas à faire la synthèse du passé, des passés dans le présent, la synthèse de la connaissance de l'Homme, nous n'existerons pas ${ }^{90}$.

Quelques semaines plus tard, Borduas précise d'ailleurs à Gauvreau le nécessaire détachement du mythe de la continuité de la culture française pré-moderne en Amérique: "Oublions, si nécessaire, les belles histoires de nos grands-mères et contribuons en toute sincérité, de tout notre poids à l'extraordinaire aventure nordaméricaine. Un avenir illimité, exceptionnel, nous attend qui vaut mieux que tous les passés de l'histoire ${ }^{91}$." Parallèlement, Borduas tient à réviser l'idéal universaliste que les factions progressistes avaient

${ }^{89}$ Borduas, Petite pierre angulaire posée dans la tourbe de mes vieux préjugés, adressée à Claude Gauvreau, Paris, novembre-décembre 1958. Écrits I, p. 543-546. Repris dans Situations, vol. 3, n 2 (mars-avril 1961), p. 60-64.

${ }^{90}$ Lettre de Borduas à David A. Gibbs, Paris, $1^{\text {er }}$ novembre 1958. Fonds privé. Écrits II, t. II, p. 1019.

${ }^{91}$ Lettre de Claude Gauvreau à Borduas, Montréal, 19 janvier 1959. Musée d'art contemporain de Montréal. Fonds Borduas (T. 128). Écrits II, t. II, p. 1041. 
caressé à Montréal en réaction au nationalisme ethnique, en élargissant sa perspective et en concevant la condition humaine en dehors des stricts référents politiques et culturels. À Claude Gauvreau, qui attribue cette nouvelle position à un mal du pays et à un retour à des valeurs rationalistes et conservatrices, il réplique:

J'ai en horreur tout nationalisme. Je reste apolitique. De se reconnaitre d'un lieu donné, d'un temps précis, est autre chose. [...] Je me suis reconnu de mon village d'abord, de ma province ensuite, Canadien français après, plus Canadien que Français à mon premier voyage en Europe, Canadien (tout court, profondément semblable à mes compatriotes) à New York, Nord-Américain depuis peu. D'ici là, je souhaite posséder la Terre entière ${ }^{92}$.

Les œuvres tardives semblent marquées à la fois par le "vertige " que procure cette perspective élargie et la difficulté qu'éprouve Borduas à trouver sa propre place dans son environnement. Réduites à des masses blanches et noires, les composantes contreviennent d'abord à la logique perceptuelle traditionnelle selon laquelle le noir sert de repoussoir au blanc qui apparait en saillie. En examinant la surface et la manière dont le pigment a été appliqué, on remarque que les masses sombres ne se trouvent ni superposées ni juxtaposées aux zones claires, mais le plus souvent à l'intérieur de celles-ci, grâce à l'effet de texture aux intervalles. À travers le jeu de leurs frontières communes, les deux valeurs tendent à conserver une ambigüité spatiale $e^{93}$. Même si, en proportion, une plus grande importance est accordée au blanc, qui tend par conséquent à être lu comme un fond, le noir semble agir comme une présence sous-jacente persistante, obscure, dont le rôle est d'éprouver, de révéler la lumière ${ }^{94}$. En réduisant leur effet tridimensionnel au seul relief de la matière ${ }^{95}$,

\footnotetext{
${ }^{92}$ Lettre de Borduas à Claude Gauvreau, Paris, 19 janvier 1959. Musée d'art contemporain de Montréal. Fonds Borduas (T. 128). Écrits II, t. II, p. 1042.

${ }^{93}$ Gagnon, Paul-Émile Borduas, p. 348. L'auteur souligne: «Ces taches noires pourront alors se lire tantôt comme des formes plaquées sur le blanc et s'en détachant, tantôt comme des trous dans le blanc, révélant encore une fois un fond infini."

${ }^{94}$ Borduas percevait d'ailleurs, chez Soulages, le même type de rapport antithétique. Cloutier-Cournoyer, Structure de l'espace pictural dans l'auvre parisienne de PaulÉmile Borduas (1955-1960), p. 14.

${ }^{95}$ Ibid., p. 5.
} 
les œuvres radicalisent alors leur mise en tension et orientent notre regard sur la dynamique dramatique des formes réalisée par leur poids plastique, leur densité et leur interaction.

Le traitement plus synthétique que dynamique que Borduas réserve toutefois aux composantes des ouvres rejoindrait ainsi davantage le courant du Color field painting dans sa dimension matérialiste. À ce titre, les œuvres s'apparentent, par leur structure comme par leur contenu, à celles de Mark Rothko, dont les parallélogrammes (multiforms) aux contours flous et superposés synthétisaient les luttes physiques et psychologiques que l'artiste avait jadis menées de part et d'autre des zones frontières, entre des univers interne et externe, d'abord à travers son expérience de migration dans l'Ouest américain (en Oregon), puis dans les zones inconnues et «souterraines" du contexte urbain new-yorkais ${ }^{96}$. Ce rapport de complémentarité n'est pas sans rappeler également la dynamique quavait exprimée Ozias Leduc au moyen de la rhétorique symboliste du passage du jour à la nuit ou du cycle saisonnier, et qui illustrait une quête de dépassement de la condition humaine, en vue d'un accomplissement spirituel ${ }^{97}$. Malgré sa distance à l'égard des valeurs religieuses, Borduas adhérait à une quête similaire d'équilibre entre l'enracinement et l'ouverture, l'immanence et la transcendance, l'espoir initial à l'acte d'accomplissement, qui traverse l'imaginaire nord-américain. Contrairement à Leduc dont les ouvres proposent une unification des opposés, moyennant leur inscription dans une temporalité cyclique et un espace mythique, ces mêmes éléments synthétisent, chez Borduas, une lutte qui semble toutefois connaître un dénouement progressif. À Judith Jasmin qui le questionne en 1957 sur l'évolution de ses œuvres, il explique: «J'ai peut-être rejoint certains de mes désirs que j'avais déjà dans ce temps-là. Je crois vous avoir parlé de l'espace, de

${ }^{96}$ Georges Roque (CNRS, Paris), "Rothko : le corps et la ville (1935-45) », Congrès international d'histoire de l'art, Montréal, 22-27 août 2004. Un aperçu des œuvres de Rothko est disponible sur le site de la National Gallery of Art de Washington: http://www.nga.gov/feature/rothko/.

${ }^{97}$ Voir Laurier Lacroix, Ozias Leduc. Une cuvre d'amour et de rêve, Musée du Québec, Musée des beaux-arts de Montréal, 1996, 318 p. 
cette appréciation en espace... et ma peinture allait vers un sentiment qui n'était pas encore mûr, qui n'était pas encore exprimable je suppose $^{98}$." Au cours de ses premiers mois d'exil aux États-Unis, Borduas exprime régulièrement, à travers sa correspondance, l'incessante valse entre l'enchantement et le désenchantement, causée par son besoin de reconnaissance, cet urgent appel au regard de l'Autre: «Euphorie et désespoir: production massive et destruction non moins massive jusqu’à ce qu'une certaine sérénité soit rejointe. Il me faut beaucoup piocher pour retrouver un peu de fraîcheur, de pureté, au fond de moi-même ${ }^{99}$." Questionnant le paradoxe et le sens de la condition d'exil chez les automatistes, Jean-Pierre Denis en note le principal enjeu: «[...] tout exil comporte de la souffrance, voire un sentiment d'échec: celui ne n'avoir pas convaincu sa propre communauté de sa valeur, de n'en avoir pas obtenu la reconnaissance ${ }^{100}$. " Cette frustration s'accompagne pourtant d'une espérance indéfectible, comme le montre Gagnon: «L'exil s'enracine dans une expérience de rejet par son milieu et est vécu comme l'occasion de merveilleuses découvertes. Il [Borduas] ne doute pas un instant que le milieu d'accueil sera un milieu plus enrichissant que celui qu'il quitte ${ }^{101}$. " Accumulant les difficultés, l'artiste finit toutefois par avouer:

Quelque chose est brisé dont j'attends la réparation: une grave contradiction dont $j$ 'attends la solution. Impossibilité de m'accepter en bloc dans le milieu. Perdu ce sens de l'unité, de la liberté. [...] Ici je suis comme perdu dans mon passé sans avoir perdu la notion du présent. C'est exécrable et j’ai hâte de me remettre à peindre et de refaire cette unité en retrouvant l'univers des possibilités nouvelles ${ }^{102}$.

\footnotetext{
${ }^{98}$ Entrevue accordée à Judith Jasmin, émission Carrefour, Radio-Canada, 1957. Retranscription dans Borduas, Refus global et autres écrits, p. 291-292.

${ }^{99}$ Lettre de Borduas à Claude Gauvreau, Provincetown, 5 août 1953. Fonds privé. Écrits I, p. 538.

${ }^{100}$ Jean-Pierre Denis, «L'exil au temps des automatistes», dans Gauvin, dir., Les automatistes à Paris, actes d'un colloque, p. 79-80.

${ }^{101}$ François-Marc Gagnon, "Les exils de Borduas ", Etc. Montréal, no 17 (février 1992), p. 10.

${ }^{102}$ Lettre de Borduas à Noël Lajoie, Paris, 26 février 1956. Fonds privé. É Erits II, p. 819.
} 
Pour Borduas, dont les espoirs sans cesse échafaudés et déchus ont fini par façonner son expérience avec le monde externe, le rapport à l'altérité et le sentiment d'éloignement en viennent à doubler l'expérience de sa propre collectivité, comme l'a remarqué JeanPierre Denis :

Borduas porte le rêve d'une nation qui cherche à rompre le cercle enchanté de son aliénation, c'est-à-dire son incapacité à exister avec force dans le regard de l'Autre, dans une perspective proprement universelle, où, certes, la part d'appartenance collective ou nationale peut et doit intervenir, mais à la condition d'être maintenue à bonne distance ${ }^{103}$.

L'histoire en a fait, comme on sait, une véritable prosopopée des enjeux collectifs et de l'accomplissement de la Révolution tranquille. Ses tentatives - conscientes ou non - de s'élever lui-même en mythe, notamment à travers ses échanges épistolaires ${ }^{104}$, dans lesquels il détaille sa pensée et ses actions, ont alors comme but de briser la paralysie qui le tiraille, mais aussi de s'incarner en modèle d'action, ou du moins, en modèle de résistance exemplaire. L'histoire a retenu la seconde option. Cette action fondatrice et créatrice sera quant à elle assumée par la génération suivante. Jean McEwen, par exemple, bénéficiera de l'influence de l'Action painting et du Color field painting par l'intermédiaire d'artistes états-uniens qu'il rencontre à Paris par l'intermédiaire de Jean-Paul Riopelle. Sensible aux champs colorés de Sam Francis, il exploite des surfaces colorées autonomes qui laissent alors la critique montréalaise dubitative. Il soutiendra d'ailleurs plus tard, à ce sujet: « Borduas, c'était encore des objets sur un fond, tandis que la peinture américaine, c'était des grands champs de peinture où pour moi il me suffisait de quelques éléments pour soutenir cette

${ }^{103}$ Denis, "Borduas hors frontière. Le One Big world", p. 35.

${ }^{104}$ Voir à sujet Gilles Lapointe, L'envol des signes. Borduas et ses lettres, Montréal, Fides, CÉTUQ, 1996, 272 p. 
vibration: la révélation ${ }^{105}$." François-Marc Gagnon a soutenu, par ailleurs, que, bien que Riopelle ait précédé son ancien professeur, au début des années 1950, dans la prise de conscience de la peinture américaine, dont plusieurs représentants étaient installés comme lui à Paris (Sam Francis, Norman Bluhm, Joan Mitchell, Al Held, Shirley Jaffe et Ellsworth Kelly, etc.), l'installation de Borduas à New York aurait été jugée par plusieurs spécialistes québécois comme un événement davantage significatif ${ }^{106}$. Nous croyons, à ce titre, que la chronologie des contacts et des influences doit être considérée à la lumière d'autres facteurs, comme celui de la dynamique relationnelle entre le sujet et son environnement, et de la résonnance de cette expérience dans l'imaginaire spatial américain. À ce propos, bien que Borduas ait pris conscience de la dimension générique et dynamique de l'espace pictural nord-américain, Riopelle en aurait exploité la réalité spatiale ainsi que l'imaginaire et les mythologies fondatrices ${ }^{107}$. En considérant dès le départ l'acte créatif comme un moyen non pas de transcrire les "nécessités internes", mais les tensions et drames de la réalité externe, le fonctionnement et le dynamisme de la nature, le « trappeur supérieur " se serait rapproché de ses compatriotes états-uniens, comme en témoignent ses encres sur papier de la seconde moitié des années 1940. Au début des années 1950, l'artiste prend le parti de libérer la forme de toute référence expressive et symbolique, en exploitant, à travers ses "mosaïques », une matière pleine et colorée, des contrastes structurants et une gestuelle assumée et contrôlée, afin

${ }^{105}$ Rapporté par Claire Gravel, «McEwen au Musée des beaux-arts », Le Devoir, 12 décembre 1987, p. C-1.

${ }^{106}$ Gagnon, «La première expérience de la peinture américaine abstraite (les années 1950) ", p. 273. Riopelle participe d'ailleurs avec plusieurs d'entre eux à l'exposition Véhémences confrontées, à la Galerie Nina Dausset, en mars 1951.

${ }^{107}$ Au sujet de la manière dont Riopelle s'est approprié le modèle fondateur du pionnier/trappeur/coureur des bois qui participait aussi bien à la création de sa persona artistique que de sa démarche esthétique, voir Vigneault, Identité et modernité dans l'art au Québec. Borduas, Sullivan, Riopelle, p. 278-315; ainsi que "Le pionnier: acteur de la frontière ", dans Andrès et Bouchard, dir., Mythes et societés des Amériques, p. 300-305. 
de maintenir l'uniformité de la surface murale. Lorsque Borduas visite l'exposition Riopelle présentée à la galerie Pierre Matisse au printemps 1955, il en livre un compte rendu partagé entre l'amertume et l'admiration: «Systématisation poussée à l'extrême limite. Efficacité totale. Égoïsme parfait où tout est en fonction de l'effet. Un art sans espoir; tout en certitude. Un art aussi sans angoisse en pleine sécurité. Il est juste que le succès couronne une telle volonté de puissance ${ }^{108}$." En fait, le parcours de Riopelle tout comme son attitude face à l'inconnu s'opposent en tout point à ceux de son ancien professeur: armé d'une bonne dose d'assurance et d'audace, de l'intuition du chasseur traquant pour éviter d'être traqué, l'artiste arbore, dans le contexte parisien, un détachement et une dégaine tout américaine. À ce titre, il assume l'individualisme et le détachement qui permettent un réel affranchissement des anciennes contraintes culturelles et sociales. Il fait d'un accomplissement dépourvu d'hésitation son moduis operandi, en conservant néanmoins un pont entre l'expression américaine et la tradition européenne. Son immersion dans le milieu parisien a aussi l'avantage de n'avoir pas été précédée d'une reconnaissance aux États-Unis, contrairement à Borduas dont le séjour prolongé à New York et le succès ont contribué à nourrir la méfiance de la critique parisienne qui doit composer avec le récent essor de l'hégémonie artistique américaine.

Malgré les déceptions accumulées, tout retour en arrière signifiait, pour Borduas, une perte des acquis et l'aveu d'un relatif échec de reconnaissance à l'étranger ${ }^{109}$. Éternel insatisfait, il entretient un

${ }^{108}$ Lettre de Borduas à Gilles Corbeil, 5 avril 1955. Musée d'art contemporain de Montréal. Fonds Borduas, double au carbone (T. 225). Écrits II, t. II, p. 750.

109 À sa sœur Jeanne, Borduas explique: « Pour moi le retour sera difficile sans perdre les avantages acquis depuis ces cinq années d'exil. Il faudrait la fortune ou la gloire soudaine pour retourner sans regrets " (Lettre à Jeanne Brisebois, Paris, 7 février 1958. Fonds privé, Écrits II, t. II, p. 971). Malgré le fait que Borduas ait connu en réalité des succès attribuables à la vente de ses œuvres, les attentes qu'il cultive à l'égard de sa reconnaissance ne semblent jamais s'assouvir. L'écrivain Michel Camus, qui comptait parmi ses proches, explique qu' " [il] était réservé et peu entreprenant pour nouer des relations avec des inconnus" ("Souveraineté 
sentiment de rejet dans ses épreuves de confrontation à l'Autre, multiplie les maladresses et les rendez-vous manqués. S'enfermant dans cette blessure, il se coupe du même coup de ceux qui, comme Riopelle, voient leur succès se solidifier. En parcourant sa correspondance, on note pourtant que le terme espoir revient sans cesse, jusqu'à devenir un véritable leitmotiv. L'artiste le traduira même à l'anglaise, comme pour mieux le rattacher à l'esprit américain: «En expectation qualifierait mieux l'état présent, où le fruit attendu compte plus que le mouvement qui le produit: restant d'ailleurs toujours un signe-témoin de ce mouvement ${ }^{110}$. " La nuance de la traduction rejoint ici plus spécifiquement la définition américaine de destiny, qui désigne non pas la soumission à un sort défini d'avance, comme le veut la définition pré-moderne et celle évoquée dans Refus global ("notre destin sembla durement fixé"), mais à l'idée judaïque d'une promesse, de ce qui doit être accompli et ne l'est pas encore, une annonce d'accomplissement, moyennant le bénéfice d'une action, un vide à remplir qui transforme les pertes de l'exil en exode fondateur. Dans les faits, Borduas, qui accorde selon son propre aveu plus d'importance au résultat qu'au chẹminement lui-même, et qui en vient même à confondre les deux, amoindrit par conséquent son pouvoir d'action et se laisse dominer par des hésitations face à la mission qu'il s'est confiée.

Cette expectation semble d'ailleurs avoir trouvé un unique accomplissement à travers les œuvres: en opacifiant progressivement le contenu expressif, et en misant sur la présence ontologique de la matière à défaut d'une réelle appropriation de l'espace, Borduas intensifie sa seule présence et les conflits d'existence qu'il partage avec

et solitude de la quête de Borduas à Paris ", dans Gauvin, dir., Les automatistes à Paris, actes d'un colloque, p. 119). Borduas attribuait cette attitude à son âge et à son état de santé: "Il me manque que l'on m'aime. Je suis trop vieux pour faire tous les premiers pas" (Rapporté par Jean Éthier-Blais, "Conversation rue Rousselet", dans Écrits I, p. 674).

${ }^{110}$ Borduas, Petite pierre angulaire posée dans la tourbe de mes vieux préjugés, texte adressé à Claude Gauvreau, Paris, novembre-décembre 1958. 
ceux qui vivent un déracinement, un nomadisme involontaire qui déstabilise en même temps qu'il renouvelle ${ }^{111}$. À ce titre, le contenu des œuvres synthétise la .condition universelle des promesses non accomplies, entre l'asile et l'exil, entre le réel et le mythe. En effectuant un retour à la forme, après avoir tenté en vain de s'en libérer, et en exacerbant sa matérialité et sa dynamique de projection dans l'espace, l'artiste assume ses luttes primordiales et en révèle le poids et l'ampleur. L'espace projeté, qu'il définit alors comme "cosmique», dans la perspective d'une participation à l'universel, est alors envisagé non plus en termes de reniement mais de potentiel à la fois absolu et matériel, comme il l'explique: «Le Cosmique ne serait plus le Chaos mais vertige d'un ordre exorbitant ${ }^{112}$. " Des œuvres comme Chatoiement (1956), Composition 64 (Monochrome gris, c1958), Composition 59 (Monochrome blanc, c1958) concrétisent ce tournant de la lumière devenue matière. En 1956, son œuvre $3+4+1$ présente également cette dynamique. Françoise Cloutier-Cournoyer et François-Marc Gagnon, qui en ont fait l'analyse, notent que les chiffres ont alors pour fonction d'orienter la lecture du regroupement des formes ${ }^{113}$. Suivant ce principe, les trois taches noires qui attirent d'abord l'attention forment un arc de cercle qui circonscrit l'amas de quatre masses noires situées dans le coin inférieur gauche. Suivant le mouvement diagonal, le regard aboutit finalement dans le coin supérieur droit où se détache un minuscule losange noir dont la pointe la plus acérée est dirigée vers le coin opposé, renforçant ainsi une impression d'élévation et d'isolement des autres composantes. Réalisée la même année, Sea Gull présente une structure similaire circonscrite par un espace davantage illusionniste: renforcé par le

${ }^{111}$ Voir Michel Maffesoli. Du nomadisme. Vagabondages initiatiques, Paris, Librairie Générale Française, Livre de Poche, coll. "Biblio Essais ", 1997, 197 p.

${ }^{112}$ Lettre publiée dans Liberté, vol: 4, n 22 (avril 1962), p. 241.

${ }^{113}$ Cloutier-Cournoyer, Structure de l'espace pictural dans l'cuvre parisienne de PaulÉmile Borduas (1955-1960), p. 34-36; Gagnon, Paul-Emile Borduas, p. 344. L'œuvre peut être consultée dans l'ouvrage de Gagnon et au site suivant: http:// www.litterature-quebecoise.org/Noirceur/Images/borduas2.jpg. 
format vertical, le mouvement est cette fois assuré par l'allègement progressif de la densité des masses noires vers le sommet ${ }^{114}$. Avec L'Étoile noire (1957) (figure 2), Borduas reprend la même dynamique que $3+4+1$ : imposant sa présence dans la partie supérieure du ciel clair de la "nuit cosmique", l'étoile exhibe sa pleine matérialité, malgré sa petite dimension. Elle se dresse sur sa pointe et s'élève dans l'espace, en s'isolant des masses noires et brunes qui s'agglutinent et imposent leur ampleur, leurs arêtes ciselées et leur stabilité. La récurrence du mouvement ascensionnel suggéré par la projection des formes dans l'espace rappelle celle qu'avait exploitée à travers l'essentiel de son œuvre Ozias Leduc, disparu deux ans plus tôt. Dans un texte de 1953, où il rend hommage au sage de Correlieu, Borduas admet que l'œuvre de ce dernier aurait marqué profondément son imaginaire:

De ma naissance à l'âge d'une quinzaine d'années ce furent les seuls tableaux qu'il me fût donné de voir. Vous ne sauriez croire combien je suis fier de cette unique source de poésie picturale à l'époque où les moindres impressions pénètrent au creux de nousmêmes et orientent à notre insu les assises du sens critique [...] Ce sera étrange pour quelques-uns d'entendre que je sois resté fidèle à l'essentiel de ces premières impressions. [...]

Je lui dois l'une des rares permissions de poursuivre mon destin. [...] Je lui dois enfin de mavoir permis de passer de l'atmosphère spirituelle et picturale de la Renaissance au pouvoir du rêve qui débouche sur l'avenir ${ }^{115}$.

À l'instar de Leduc, qui avait privilégié un long et lent cheminement, Borduas envisage désormais l'enracinement non plus comme un cantonnement, mais comme un axis mundi, un correlieu ou un home anglo-saxon qui réunit à la fois l'idée d'un chez-soi et du pays natal. Cette réalité lui permet alors d'ouvrir à une expérience existentielle élargie, et d'embrasser le réel pour mieux le transcender. Ce même

${ }^{114}$ Cloutier-Cournoyer, Structure de l'espace pictural dans l'ouvre parisienne de PaulÉmile Borduas (1955-1960), p. 53; Gagnon, Paul-Émile Borduas, p. 350.

115 Paul-Émile Borduas, "Quelques pensées sur l'œuvre d'amour et de rêve de M. Ozias Leduc", Canadian Art, vol. 10, n 4 (été 1953), p. 158-161, 168. 


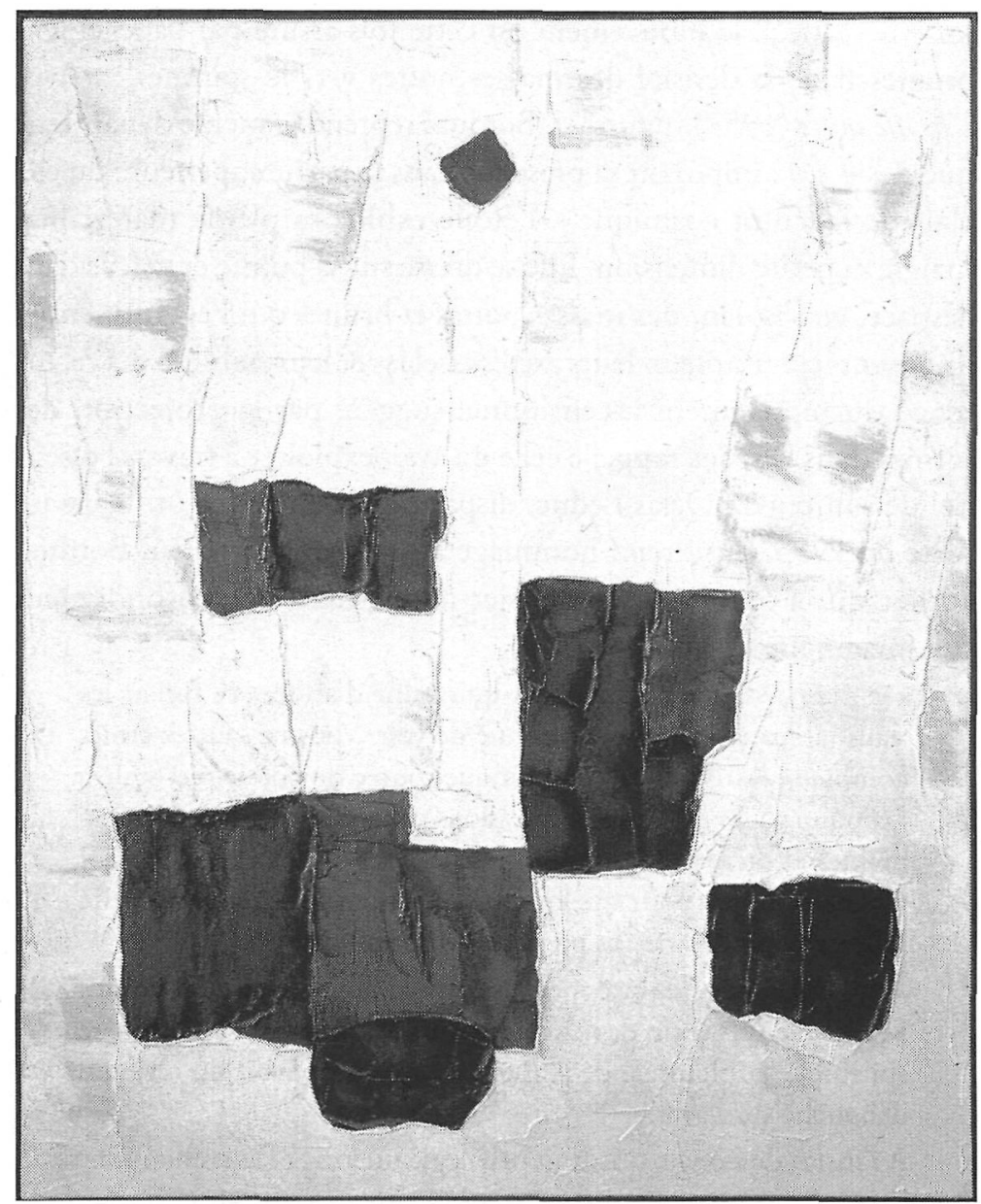

Figure 2 - Paul-Émile Borduas, Létoile noire, 1957; huile sur toile, $162,5 \mathrm{~cm} \mathrm{x}$ $129,5 \mathrm{~cm}$. Musée des beaux-arts de Montréal (Don de M. et de $\mathrm{M}^{\mathrm{me}}$ Gérard Lortie; $\mathrm{n}^{\circ}$ 1960.1238; photo: Denis Farley, MBAM). 
idéal, qui a contribué à nourrir de nombreux mythes américains comme celui de la Terre Promise, de la rédemption par le transit initiatique, et de l'amélioration des conditions de vie ${ }^{116}$, s'est trouvé renforcé, chez Borduas, à mesure qu'évoluaient ses déplacements. De ses premiers réflexes d'arrachement à son milieu, qui lui a d'abord permis de rejoindre des modèles périphériques susceptibles d'exprimer ses "nécessités intérieures", l'artiste réamorce par la suite ses élans, plus radicaux, afin d'épurer les zones troubles qui le tenaillent, et de mener une lente réconciliation des oppositions. Ce processus se réalise au prix d'une élimination progressive des contenus strictement émotionnels et sensitifs, et au profit d'une prise de conscience élargie de sa condition dans l'environnement, de son mouvement à travers lui, entre le vertige du vide et un éveil libérateur. L'organisation des composantes des œuvres, tout comme les titres évocateurs de réalités territoriales, révèlent alors ces mouvements de transit et de devenir. La prise de conscience de l'artiste du pouvoir de l'action à travers l'espace lui donne finalement accès à la dimension mythique, laquelle confere à l'environnement une charge culturelle et un enracinement significatif, et permet de transcender les repères traditionnels et le clivage séculaire entre les appartenances américaine et européenne. Il aurait sans doute fallu quelques années encore pour que les conflits qui habitaient l'artiste se muent en une réelle rencontre avec l'Autre, au-delà du besoin de s'en faire reconnaître, afin d'assouvir les besoins d'ouverture et de renouvellement qui lui sont restés vitaux. L'idée qu'il se faisait alors de l'Amérique, de son point de vue européen, lui aurait fourni un sentiment à la fois d'absolu et de familier.

${ }^{116}$ Patrick Imbert, "Les trois $\mathrm{R}$ - ruptures, routes, réussite - dans les Amériques: entre l'oubli et la promesse", dans Andrès et Bouchard, dir., Mythes et sociétés des Amériques, p. 139-172. 